\title{
Nonextensive hydrodynamics for relativistic heavy-ion collisions
}

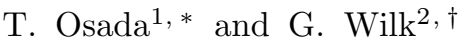 \\ ${ }^{1}$ Theoretical Physics Lab., Faculty of Knowledge Engineering, \\ Musashi Institute of Technology, Setagaya-ku, Tokyo 158-8557, Japan \\ ${ }^{2}$ The Andrzej Sottan Institute for Nuclear Studies, Hoża 69, 00681, Warsaw, Poland
}

(Dated: September 10, 2018)

\begin{abstract}
The nonextensive one-dimensional version of a hydrodynamical model for multiparticle production processes is proposed and discussed. It is based on nonextensive statistics assumed in the form proposed by Tsallis and characterized by a nonextensivity parameter $q$. In this formulation the parameter $q$ describes some specific form of local equilibrium which is characteristic for the nonextensive thermodynamics and which replaces the local thermal equilibrium assumption of the usual hydrodynamical models. We argue that there is correspondence between the perfect nonextensive hydrodynamics and the usual dissipative hydrodynamics. It leads to simple expression for dissipative entropy current and allows for predictions for the ratio of bulk and shear viscosities to entropy density, $\zeta / s$ and $\eta / s$, to be made.

PACS numbers: 24.10.Nz, 25.75.-q
\end{abstract}

\section{INTRODUCTION}

Multiparticle production experiments are our main source of information on multiparticle production processes in which the initial kinetic energy of two projectiles is to a large extent converted into a multitude of observed secondaries. This is especially true in the case of heavy ion collisions in which one expects the formation of a new hadronic state of matter, the Quark Gluon Plasma (QGP) 1]. Such processes call for some form of statistical approach, which is usually based on the BoltzmannGibbs (BG) statistics. On the other hand, in the case of a multiparticle production process, conditions leading to BG statistics are satisfied only approximately at best. This is because, among other things, hadronizing systems experience strong intrinsic fluctuations and long range correlations [2, 3], which can be interpreted as signals of some dynamical, nonequilibrium effects showing up[4]. It is therefore difficult to expect the occurrence of the usual (local) thermal equilibrium, instead one has some kind of stationary state. It turns out that these phenomena can be incorporated, at least to some extent and without going into deeper dynamical considerations concerning the sources of such fluctuations, in the formalism of the nonextensive statistics (which we shall apply here in the manner proposed by Tsallis, see [5]) in the form of a more general equilibrium summarily described by a single parameter $q[6,6,8,9]$. This parameter characterizes the corresponding Tsallis entropy, $S_{q}$, which in such approach replaces the usual BG entropy to which it converges for $q \rightarrow 1$. Because such systems are in general nonextensive, the parameter $q$ is usually called a nonextensivity parameter.

Such approach has been successfully applied to mul-

\footnotetext{
*Electronic address: osada@ph.ns.musashi-tech.ac.jp
}

† Electronic address: wilk@fuw.edu.pl tiparticle production processes, both by using nonextensive versions of the respective distribution functions 10, 11, 12, 13, 14, 15, 16], or by deriving such distributions from the appropriately modified nonextensive version of the Boltzmann transport equation [6, 7, 9, 17, 18]. In both cases, this amounts to replacing the usual exponential factors by their $q$-exponential equivalents,

$$
\begin{gathered}
P_{\mathrm{BG}}(E)=\exp (-E / T) \Longrightarrow P_{q}(E)=\exp _{q}(-E / T) \\
=[1-(1-q) E / T]^{1 /(1-q)} .
\end{gathered}
$$

Notice that $P_{\mathrm{BG}}(E)=P_{q=1}(E)$. In all these applications one finds that $q>1$. It represents effect of some intrinsic fluctuations existing in the hadronizing system and revealing themselves as fluctuations of its temperature or of the mean multiplicity of secondaries 57]. Generically one has that 2]

$$
q=1+\frac{<(1 / T)^{2}>}{<(1 / T)>^{2}},
$$

where, depending on the kind of process considered, $T$ can be replaced by some other variable [13, 14, 15]. Because different observables are sensitive to different kinds of fluctuations, it is natural to expect that they are described by different values of the parameter $q$ [14]. For example, single particle, one-dimensional distributions in longitudinal phase-space, like $d N / d y$, are most sensitive to fluctuations of the mean multiplicity $\langle n\rangle$ of secondaries [13] and are described by the nonextensivity parameter $q=q_{\mathrm{L}}$ of the order of $q_{\mathrm{L}}-1 \sim 0.1 \div 0.2$ [58]. However, distributions in transverse momenta, $d N / d p_{\mathrm{T}}$, which are believed to probe the local thermal equilibrium of the hadronizing system (assuming that such a phenomenon indeed occurs) and serve as a source of information on the temperature $T$ of the hadronizing system, are very sensitive to fluctuations of this temperature represented by the nonextensivity parameter $q=q_{\mathrm{T}}$ [15]: changes as small as $q_{\mathrm{T}}-1 \sim 0.01 \div 0.05$ already substantially affect the resultant $p_{\mathrm{T}}$ spectra [59] Notice that data indicate 
that $q_{\mathrm{L}}-1 \gg q_{\mathrm{T}}-1$, i.e., fluctuations governed by $q_{\mathrm{L}}$ are dominant and therefore for the whole system $q \sim q_{\mathrm{L}}$ [14.

Among statistical approaches to the multiparticle production processes a specially important role is played by hydrodynamical models [21], which so far are all based on the BG statistics. The existing general nonextensive version of fluid dynamics discussed in [22] is not suitable for applications to multiparticle production processes (among other things because of its non covariant formulation). We would like to fill this gap and present a fully covariant hydrodynamical model based on $q$-statistics which can be applied to a multiparticle production processes, especially to relativistic heavy-ion collisions. Because of exploratory character of our paper we limit ourselves to the $(1+1)$ dimensional case only and confront our results with rapidity and transverse momenta distributions obtained recently at RHIC leaving the most detailed studies of all aspects of available experimental data for future investigations.

The hydrodynamical model of multiparticle production means, in fact, a number of separate problems connected with the consecutive steps of the collision process: the choice of initial conditions summarizing the preparatory stage of collision (it should end in some form of local thermal equilibrium), the choice of equation of state (EoS) of the quark-gluon and/or hadronic matter being equilibrated, further hydrodynamical evolution of this matter assumed to form a kind of fluid, final conversion of this fluid into observed secondaries. Because dynamical factors underlying each step are different the resulting fluctuation patterns can also differ, presumably leading to the parameter $q$ changing during the collision process. However, in the present study we shall restrict ourself only to the case of nonextensive parameter $q$ remaining the same in the whole collision process [60].

Recently there is renewed interest in dissipative hydrodynamical models 223, 24, 25, 26, 27, 28, 29, 30, 31, 32], which is prompted by the apparent success of hydrodynamical models in describing data obtained at RHIC $[33,34,35]$ and by the recent calculations of transport coefficients of strongly-interacting quark-gluon system using the AdS/CFT correspondence [36]. The question addressed is whether, and under which circumstances, dissipative hydrodynamics is really needed and how it should be applied. The reason is that formulation of the relativistic hydrodynamic equations for dissipative fluid, suffer from ambiguities in the form they are written [27], unphysical instability of the equilibrium state in the first order theory [28] and the loss of causality in the first order equation approach [31], to mention a few. In this work we argue that there is a link between dissipative hydrodynamics ( $d$-hydrodynamics) and nonextensive hydrodynamics ( $q$-hydrodynamics) we are proposing, which we call nonextensive/dissipative correspondence (NexDC). In particular, in Section $\mathrm{V}$ we demonstrate that it is possible to write some of the corresponding transport coefficients of the produced matter (believed to be quark-gluon plasma (QGP)) as (implicit) functions of the nonextensivity parameter $q$. The merit of using the NexDC is that one can formulate and solve the $q$-hydrodynamic equations of perfect nonextensive hydrodynamics (or perfect $q$-hydrodynamics) in analogous way as for the usual perfect hydrodynamics, which seems to be a priori much easier task. Although this does not fully solve the problems of $d$-hydrodynamics, nevertheless it allows us to extend the usual perfect fluid approach (using only one new parameter $q$ ) well behind its usual limits, namely toward the regions reserved for dissipative approach only.

The paper is organized as follows. We start in Section III with a short reminder of the nonextensive version of kinetic theory from which nonextensive hydrodynamics is derived in Section [II] Section[V]contains examples of comparisons with experimental data, whereas in Section [V we discuss the possible physical meaning of the proposed $q$-hydrodynamics. We end with Section VI which contains our conclusions and summary. Some specialized topics and derivations are presented in Appendices $\mathrm{A}, \mathrm{B}$. C] and D.

\section{RELATIVISTIC NONEXTENSIVE KINETIC THEORY}

Following [17] we start with nonextensive version of Boltzmann equation (the metric used is: $g^{\mu \nu}=$ $\operatorname{diag}(1,-1,-1,-1))$,

$$
\begin{aligned}
p^{\mu} \partial_{\mu} f_{q}^{q}(x, p) & =C_{q}(x, p) \\
C_{q}(x, p) & =\frac{1}{2} \int \frac{d^{3} p_{1}}{p_{1}^{0}} \frac{d^{3} p^{\prime}}{p^{0}} \frac{d^{3} p_{1}^{\prime}}{p_{1}^{\prime 0}}\left\{h_{q}\left[f_{q}^{\prime}, f_{q 1}^{\prime}\right] W\left(p^{\prime}, p_{1}^{\prime} \mid p, p_{1}\right)-h_{q}\left[f_{q}, f_{q 1}\right] W\left(p, p_{1} \mid p^{\prime}, p_{1}^{\prime}\right)\right\} .
\end{aligned}
$$

Here $f_{q}(x, p)$ and $C_{q}(x, p)$ are $q$-versions of the, respectively, corresponding phase space distribution function and the $q$-collision term in which $W\left(p, p_{1} \mid p^{\prime}, p_{1}^{\prime}\right)$ is the transition rate between the two particle state with initial four-momenta $p$ and $p_{1}$ and some final state with four-momenta $p^{\prime}$ and $p_{1}^{\prime}$ whereas $h_{q}\left[f_{q}, f_{q 1}\right]$ is the corre- 
lation function related to the presence of two particles in the same space-time position $x$ but with different fourmomenta $p$ and $p_{1}$, respectively. Notice two distinct features of Eq. (3a): $(i)$ it applies to $f_{q}^{q}\left(=\left(f_{q}\right)^{q}\right)$ rather than to $f_{q}$ itself and $(i i)$ in $C_{q}$ one assumes a new, $q$ generalized, version of the Boltzmann molecular chaos hypothesis [6, 7, 8, 9, 17, 37] according to which

$$
h_{q}\left[f_{q}, f_{q 1}\right]=\exp _{q}\left[\ln _{q} f_{q}+\ln _{q} f_{q 1}\right]
$$

where $\exp _{q}(X)=[1+(1-q) X]^{1 /(1-q)}$ and $\ln _{q}(X)=$ $\left.\left[X^{(1-q)}-1\right] /(1-q)\right)$. Eq. (4) is our central point, it amounts to assuming that, instead of the strict (local) equilibrium, a kind of stationary state is being formed, which also includes some interactions (see [6, 7, [8, 9]).

With such correlation function one finds that divergence of the entropy current, which we define as

$$
s_{q}^{\mu}(x) \equiv-k_{\mathrm{B}} \int \frac{d^{3} p}{(2 \pi \hbar)^{3}} \frac{p^{\mu}}{p^{0}}\left\{f_{q}^{q}(x, p) \ln _{q} f_{q}(x, p)-f_{q}(x, p)\right\},
$$

is always positive at any space-time point,

$$
\partial_{\mu} s_{q}^{\mu}(x) \geq 0
$$

(this fact is equivalent to demonstrating the validity of the relativistic local $H$-theorem when using this $q$ entropy current).

To get explicit form of the distribution functions $f_{q}(x, p)$ we proceed now as follows [17]. At first, using momentum conservation condition in two particle collisions, $p^{\mu}+p_{1}^{\mu}=p^{\prime \mu}+p_{1}^{\prime}{ }^{\mu}$, we form following collision invariant:

$$
F[\psi]=\int \frac{d^{3} p}{p^{0}} \psi(x, p) C_{q}(x, p) \equiv 0,
$$

where $\psi(x, p)=a(x)+b_{\mu}(x) p^{\mu}$ with arbitrary functions $a(x)$ and $b_{\mu}(x)$. We assume here that the correlation function $h_{q}$ is symmetric and positive, $h_{q}\left[f, f_{1}\right]=h_{q}\left[f_{1}, f\right] \geq 0$, and that detailed balance holds, $W\left(p, p_{1} \mid p^{\prime}, p_{1}^{\prime}\right)=W\left(p^{\prime}, p_{1}^{\prime} \mid p, p_{1}\right)$. For $a(x) \equiv 0$ and $b_{\mu}(x)=$ constant one gets the $q$-version of the local energy-momentum conservation [17],

$$
\partial_{\nu} \mathcal{T}_{q}^{\mu \nu}(x)=0
$$

with a nonextensive energy-momentum tensor defined by

$$
\mathcal{T}_{q}^{\mu \nu}(x) \equiv \frac{1}{(2 \pi \hbar)^{3}} \int \frac{d^{3} p}{p^{0}} p^{\mu} p^{\nu} f_{q}^{q}(x, p)
$$

At the same time for $a(x)=$ constant and $\left.b_{\mu}(x) \equiv 0\right)$ one gets [17]

$$
\partial_{\mu} \int \frac{d^{3} p}{(2 \pi \hbar)^{3}} \frac{p^{\mu}}{p^{0}} f_{q}^{q}(x, p)=0
$$

this implies that ( $d \Omega$ stands for the corresponding phase space volume element)

$$
\frac{d}{d t} \int d \Omega f^{q}(x, p)=0
$$

i.e., that the normalization $Z_{q} \equiv \int d \Omega f_{q}^{q}(x, p)$ is conserved as well [61]. Since the divergence of the $q$-entropy current can also be expressed via collision invariant,

$$
\partial_{\mu} s_{q}^{\mu}=\frac{1}{(2 \pi \hbar)^{3}} F\left[\ln _{q} f_{q}(x, p)\right]
$$

demanding that $\partial_{\mu} s_{q}^{\mu}(x) \equiv 0$ one finally obtains

$$
f_{q}(x, p)=\left[1+(1-q)\left(a(x)+b_{\mu}(x) p^{\mu}\right)\right]^{1 /(1-q)},
$$

which represents the distribution function in a stationary state. Setting $a(x)=0$ and $b^{\mu}(x)=-u_{\alpha}^{\mu}(x) / k_{\mathrm{B}} T_{q}(x)$ (where $T_{q}(x)$ is the temperature function [62] ) one obtains the well known (unnormalized) Tsallis distribution function,

$$
\begin{aligned}
f_{q}(x, p) & =\left[1-(1-q) \frac{p_{\mu} u_{q}^{\mu}}{k_{B} T_{q}(x)}\right]^{1 /(1-q)} \\
& \equiv \exp _{q}\left[-\frac{p_{\mu} u_{q}^{\mu}(x)}{k_{B} T_{q}(x)}\right]
\end{aligned}
$$

where $u_{q}^{\mu}(x)$ should be regarded as a hydrodynamical flow four vector (hereafter we use the convention that $\hbar=$ $\left.k_{\mathrm{B}}=c=1\right)$.

We shall now assume that the $q$-modified energymomentum tensor $\mathcal{T}_{q}^{\mu \nu}$ can be decomposed in the usual way in terms of the $q$-modified energy density and pressure, $\varepsilon_{q}$ and $P_{q}$, by using the $q$-modified flow $u_{q}^{\mu}$ (such that for $q \rightarrow 1$ it becomes the usual hydrodynamical flow $u^{\mu}$ and in the rest frame of the fluid $\left.u_{q}^{\mu}=(1,0,0,0)\right)$,

$$
\begin{aligned}
\mathcal{T}_{q}^{\mu \nu} & =\left(\varepsilon_{q}+P_{q}\right) u_{q}^{\mu} u_{q}^{\nu}-P_{q} g^{\mu \nu} \\
& =\varepsilon_{q} u_{q}^{\mu} u_{q}^{\nu}-P_{q} \Delta_{q}^{\mu \nu} .
\end{aligned}
$$

where $\Delta_{q}^{\mu \nu} \equiv g^{\mu \nu}-u_{q}^{\mu} u_{q}^{\nu}$. Denoting $e \equiv p^{0} / T, z \equiv m / T$, with $g$ being degeneracy factor depending on the type of particles composing our fluid, one gets that in its rest frame (or in $q$-equilibrium) 


$$
\begin{aligned}
\varepsilon_{q} & \equiv u_{q \mu} \mathcal{T}_{q}^{\mu \nu} u_{q \nu} \quad=\frac{g T_{q}^{4}}{2 \pi^{2}} \int d e \sqrt{e^{2}-z^{2}} e^{2}[1-(1-q) e]^{q /(1-q)}, \\
P_{q} \equiv-\frac{1}{3} \mathcal{T}_{q}^{\mu \nu} \Delta_{q \mu \nu} & =\frac{g T_{q}^{4}}{2 \pi^{2}} \int d e \sqrt{e^{2}-z^{2}} e[1-(1-q) e]^{1 /(1-q)}, \\
s_{q} & \equiv s_{q}^{\mu} u_{q \mu} \quad=\frac{g T_{q}^{3}}{2 \pi^{2}} \int d e \sqrt{e^{2}-z^{2}} e\left\{e[1-(1-q) e]^{q /(1-q)}+[1-(1-q) e]^{1 /(1-q)}\right\}
\end{aligned}
$$

(notice that for $q<1$ the integration range is limited to $z \leq \epsilon \leq 1 /(1-q)$ in order to keep the integrand positive). It is straightforward to check that, in the baryon free case to which we shall limit ourselves here,

$$
T_{q} s_{q}=\varepsilon_{q}+P_{q}
$$

and

$$
\frac{d P_{q}}{d T_{q}}=s_{q}
$$

i.e., that the usual thermodynamic relations also holds for the $q$-modified quantities.

\section{THE NONEXTENSIVE HYDRODYNAMICAL MODEL - $q$-HYDRODYNAMICS}

\section{A. Equations of nonextensive flow - $q$-flow}

Our starting point in formulating the $q$ hydrodynamical model is Eq. (8) with energy-momentum tensor $\mathcal{T}_{q}^{\mu \nu}$ given by Eq. (91). Because, due to the $q$ version of thermodynamical relations, Eq. (17), in our case Eq. (8) also implies conservation of $q$-entropy,

$$
\partial_{\mu} s_{q}^{\mu}=0
$$

with $s_{q}^{\mu}(x)$ defined by Eq. (5), which can also be written as

$$
s_{q}^{\mu}(x)=s_{q}(x) u_{q}^{\mu}(x),
$$

we have only one general equation which, when written using general coordinates and covariant derivatives [63], takes the form:

$$
\mathcal{T}_{q ; \mu}^{\mu \nu}=\left[\left(\varepsilon_{q}+P_{q}\right) u_{q}^{\mu} u_{q}^{\nu}-P_{q} g^{\mu \nu}\right]_{; \mu}=0
$$

This means that we are dealing here with perfect $q$ hydrodynamics.

Before proceeding further some specific points of $q$ hydrodynamics, not mentioned in the general derivation oresented in Section II must be kept in mind. At first notice that, whereas in the usual perfect hydrodynamics (based on BG statistics) entropy is conserved in hydrodynamical evolution both locally and globally, in the nonextensive approach it is conserve only locally, cf., Eq. (18). The total entropy of the whole expanding system is not conserved, because for two volumes $V_{1,2}$ one finds that

$$
S_{q}^{\left(V_{1}\right)}+S_{q}^{\left(V_{2}\right)} \neq S_{q}^{\left(V_{1} \oplus V_{2}\right)}
$$

where $S_{q}^{(V)}$ are the corresponding total entropies. Although, strictly speaking, the hydrodynamical model does not require global entropy conservation but only its local conservation, the above feature of $q$-hydrodynamics should be always remembered (the consequences of this fact will be discussed in more detail in Section $\mathrm{V}$ ). Second point concerns the causality problem. To guarantee that hydrodynamics makes sense, there should exists some spacial scale $L$ such that volume $L^{3}$ contains enough particles composing our fluid. However, in case when there are some fluctuations and/or correlations with some typical correlation length $l$, for which we expect that $l>L$, one has to use nonextensive entropy $S_{q}^{\left(L^{3}\right)}$ (cf. Eq. (21)) and its defined locally density $s_{q}(x)=S_{q}^{\left(L^{3}\right)} / L^{3}$. When formulating the corresponding $q$-hydrodynamics one takes, as usual, limit $L \rightarrow 0$, in which case explicit dependence on the scale $L$ vanishes whereas the correlation length leaves its imprint as parameter $q$. In this sense perfect $q$-hydrodynamics can be considered as preserving causality and nonextensivity $q$ is then related with the correlation length $l$. One can argue that very roughly that $q \sim l / L_{\text {eff }} \geq 1$, where $L_{\text {eff }}$ is some effective spacial scale of the $q$-hydrodynamics. Note here that if the correlation length $l$ is compatible with the scale $L_{\text {eff }}$, i.e., $l \approx L_{\text {eff }}$, one recovers condition of the usual local thermal equilibrium and in this case the $q$-hydrodynamics reduces to the usual (BG) hydrodynamics.

Let us now continue our presentation. When contracted with the velocity $u_{q \nu}$ or with the projection tensor $\Delta_{q \lambda \nu} \equiv g_{\lambda \nu}-u_{q \lambda} u_{q \nu}$ it leads to the following two equations:

$$
\begin{aligned}
u_{q}^{\mu} \partial_{\mu} \varepsilon_{q}+\left(\varepsilon_{q}+P_{q}\right) u_{q ; \mu}^{\mu}-P_{q} u_{q \nu} g_{; \mu}^{\mu \nu} & =0 \\
\left(\varepsilon_{q}+P_{q}\right) & u_{q}^{\mu} \Delta_{q \lambda \nu} u_{q ; \mu}^{\nu} \\
& -\Delta_{q \lambda \nu} \partial^{\nu} P_{q}-P_{q} \Delta_{q \lambda \nu} g_{; \mu}^{\mu \nu}=0 .
\end{aligned}
$$

These are the equations to be solved now for the $(1+1)$ dimensional case. We shall assume longitudinal expansion only and introduce proper time $\tau$ and the space-time 
rapidity $\eta$ :

$$
\begin{aligned}
\tau & \equiv \sqrt{t^{2}-z^{2}} \\
\eta & \equiv \frac{1}{2} \ln \frac{t+z}{t-z} .
\end{aligned}
$$

The corresponding metric tensor in this $(\tau-\eta)$ space is $g^{\mu \nu}=\operatorname{diag}\left(1,-\frac{1}{\tau^{2}}\right)$. The corresponding four velocity of our fluid can be expressed by the local fluid rapidity $\alpha_{q}(x)$ as

$$
u_{q}^{\mu}(x)=\left[\cosh \left(\alpha_{q}-\eta\right), \frac{1}{\tau} \sinh \left(\alpha_{q}-\eta\right)\right] .
$$

In this case Eq. (22) reduces to (here $\left.v_{q} \equiv \tanh \left(\alpha_{q}-\eta\right)\right)$

$$
\frac{\partial \varepsilon_{q}}{\partial \tau}+\frac{v_{q}}{\tau} \frac{\partial \varepsilon_{q}}{\partial \eta}+\left(\varepsilon_{q}+P_{q}\right)\left\{v_{q} \frac{\partial \alpha_{q}}{\partial \tau}+\frac{1}{\tau} \frac{\partial \alpha_{q}}{\partial \eta}\right\}=0
$$

whereas Eq. (23) reduces to the $q$-generalized relativistic Euler equation (cf. Appendix $\mathrm{A}$ for details):

$$
\left(\varepsilon_{q}+P_{q}\right)\left\{\frac{\partial \alpha_{q}}{\partial \tau}+\frac{v_{q}}{\tau} \frac{\partial \alpha_{q}}{\partial \eta}\right\}+v_{q} \frac{\partial P_{q}}{\partial \tau}+\frac{1}{\tau} \frac{\partial P_{q}}{\partial \eta}=0 .
$$

To solve these equations one needs additional input in terms of EoS, $P_{q}=P_{q}\left(\varepsilon_{q}\right)$, and the choice of boundary conditions, which we set as $v_{q}=0$ at $\eta=0$ (because of the symmetry $\alpha \equiv 0$ ). At $\eta=0$ Eqs. (22) and (23) reduce to

$$
\begin{aligned}
& \frac{\partial \varepsilon_{q}}{\partial \tau}=-\left.\frac{\varepsilon_{q}+P_{q}}{\tau} \frac{\partial \alpha}{\partial \eta}\right|_{\eta=0} \\
& \left.\frac{\partial P_{q}}{\partial \eta}\right|_{\eta=0}=0
\end{aligned}
$$

\section{B. Nonextensive Equation of State - $q$-EoS}

The next important ingredient of any hydrodynamical model is an Equation of State (EoS) defining a relation between the pressure and the energy density, which depends on the properties of the hadronic matter under consideration. In this work we shall only work with EoS for the relativistic pion gas (with $m_{\pi}=0.14 \mathrm{GeV}$ ) without considering different phases of hadronic matter as in [39]. The pressure $P_{q}$ and the energy density $\varepsilon_{q}$ can be connected in the form of EoS, $P_{q}\left(\varepsilon_{q}\right)$, using Eqs.(16a) and (16b). However, differently than in the usual cases of $q=1$, the additional freedom represented by the nonextensivity parameter $q$ makes $P_{q}=P_{q}\left(\varepsilon_{q}\right)$ ambiguous and one has to additionally specify the possible variations of the parameter $q$ during the evolution process. In what follows, we shall assume that the parameter $q$ remains fixed during the the whole evolution of our hadronic fluid. We therefore get different EoS for different (but fixed) values of the parameter $q$, examples of which are shown in Figure 1. It displays the ratio $P_{q} / \varepsilon_{q}$ as a function of energy density $\varepsilon_{q}$ for different values of $q=1.0,1.1$ and

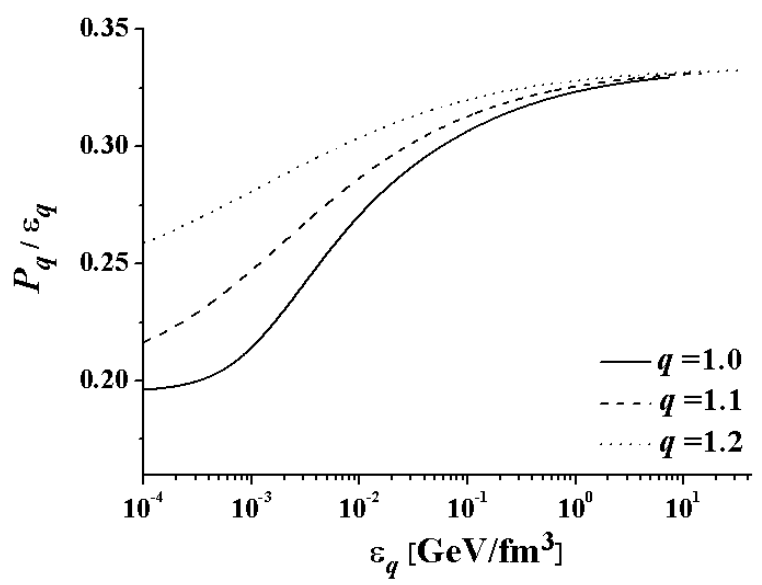

FIG. 1: Illustration of EoS for the relativistic nonextensive pionic gas $(m=0.14 \mathrm{GeV})$ : the ratio $P_{q} / \varepsilon_{q}$ is plotted as function of energy density $\varepsilon_{q}$ for different nonextensivity parameters $q$. Notice that the $q$ dependence of EoS shows mainly at low and very low energy densities.

1.2 ; the temperature $T_{q}$ was varied in the range $0.1 \div 500$ $\mathrm{MeV}$. It turns out that the $q$-dependence is confined only to the very low energy density region (supporting therefore the previous results on this matter in $[17,40]$ ). In the region of interest, $\varepsilon_{q} \sim 0.1 \div 5.0 \mathrm{GeV} / \mathrm{fm}^{3}$, the changes are very small and rapidly vanish with increasing $\varepsilon$.

\section{Nonextensive initial conditions - $q$-initial conditions}

To solve equations of $(1+1) q$-hydrodynamics one has to decide on the initial conditions from which the hydrodynamical expansion starts. They must contain some form of the local thermal equilibrium, which - as we assume - is established during the collision process. According to recent estimations this can happen very rapidly, already in the first $1 \mathrm{fm}$ of expansion, if caused by some violent, nonperturbative mechanisms operating at this stage [64]. It is thus natural to expect that there must also exist some intrinsic fluctuations already present in this preparatory stage of collision process which, according to our philosophy, should be accounted for by the same $q$-statistical approach as that used to form the $q$ hydrodynamics. Following [39, 43] we shall use Gaussian initial conditions interpolating between two extreme situations, the one described by the Bjorken scaling type model 44 and the other corresponding to the Landau Model [45], but we shall modify them accordingly by changing $\exp (X)$ to $\exp _{q}(X)$ (as in Eq. (4), it reduces to the usual Gaussian of [39] for $q=1$ ). As in [39] initial conditions are imposed for the energy density $\varepsilon_{q}$ ex- 
pressed as a function of rapidity $\eta$ :

$$
\varepsilon_{q}\left(\tau_{0}, \eta\right)=\varepsilon^{(\mathrm{in})} \exp _{q}\left[-\frac{\eta^{2}}{2 \sigma^{2}}\right]
$$

In what follows we shall also require that the $q$-fluid and the space-time rapidities coincide at $\tau_{0}$,

$$
\alpha_{q}\left(\tau_{0}, \eta\right)=\eta
$$

In all calculations presented in this paper we shall assume for simplicity that $\varepsilon_{q}$ and $\alpha_{q}$ are independent of the transverse coordinate. However, the remaining two parameters, $\varepsilon^{(\text {in) }}$ and $\sigma$ are not independent because one has to reproduce the total energy $E_{\text {tot }}$ allocated to the fluid which is fixed by the conditions of the experiment,

$$
E_{\mathrm{tot}}=\pi A_{\mathrm{T}}^{2} \tau_{0} \int d \eta \varepsilon_{q}\left(\tau_{0}, \eta\right)
$$

where $A_{\mathrm{T}}$ is the transverse size of the fluid, $\tau_{0}$ is the initial proper time $\tau$ when the fluid starts to expand. The $E_{\text {tot }}$ can be obtained knowing the mean number of participating nucleons $N_{\text {part }}$ and the total energy loss per participating nucleon $\Delta E[65]$

$$
E_{\text {tot }}=N_{\text {part }} \Delta E \text {. }
$$

The possible initial conditions vary therefore between two extremal situations (cf., Fig. 2):

(i) The width $\sigma$ in Eq. (29) is assumed to be fixed and kept constant, but its distribution to vary by changing $\varepsilon^{(\mathrm{in})}$ to reproduce the fixed total energy $E_{\text {tot }}$ when the nonextensive parameter $q$ changes.

(ii) The maximum energy density $\varepsilon^{(\mathrm{in})}$ in $\mathrm{Eq} \cdot(29)$ is assumed to be fixed and kept constant, but its distribution to vary by changing $\sigma$ to reproduce the fixed total energy $E_{\text {tot }}$ when the nonextensive parameter $q$ changes.

We would like to stress at this point that such $q$ dependent initial conditions introduce a completely new element to hydrodynamical models, not discussed previously. The real situation will interpolate in an a priori unknown manner between these two extremes, therefore, in what follows, we shall restrict ourself only to them. As one can see in Fig. 2 whereas the first extreme introduces sizable $q$-dependence, the second one leads to only minor effects. In both cases, increasing the value of $q$ results, as expected [13], in the enhancement of tails for large values of $\eta$. Following [39] our calculations were performed for $\mathrm{Au}+\mathrm{Au}$ collisions at $\sqrt{s_{\mathrm{NN}}}=200 \mathrm{GeV}$, using results reported by the BRAHMS experiment [46], with $E_{\text {tot }}=26.1 \mathrm{TeV}, N_{\text {part }}=357, \Delta E=73 \pm 6 \mathrm{GeV}$ and with $A_{\mathrm{T}}=6.5 \mathrm{fm}, \tau_{0}=1.0 \mathrm{fm}$, see Table【 In Fig. 3 are shown, the initial conditions for the, respectively, energy density $\varepsilon_{q}\left(\tau_{0}, \eta\right)$, entropy density $s_{q}\left(\tau_{0}, \eta\right)$ and temperature $T_{q}\left(\tau_{0}, \eta\right)$ in the case of $\sigma=1.25$ fixed (panels (a)-(c)) and $\varepsilon^{(\mathrm{in})}=27.8 \mathrm{GeV} / \mathrm{fm}^{3}$ fixed (panels
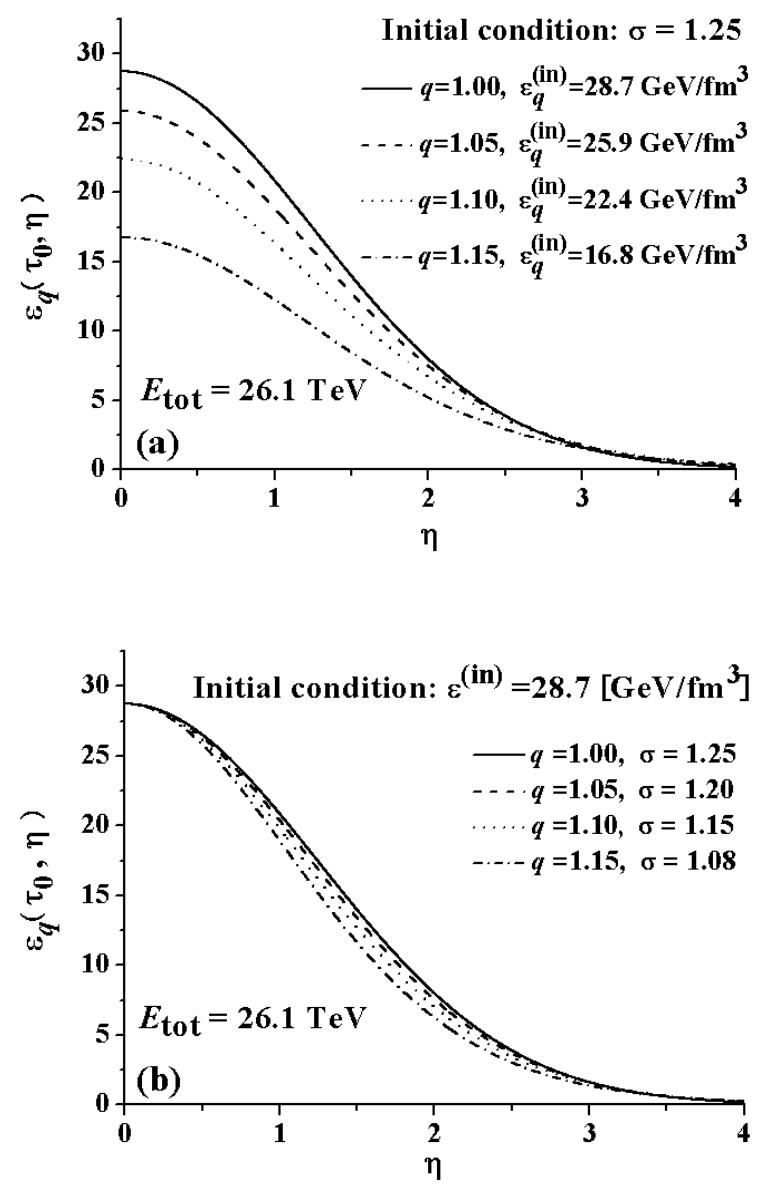

FIG. 2: Examples of two different types of initial conditions: (a) - type $(i)$ initial conditions with fixed $\sigma=1.25$ and $\varepsilon^{\text {(in) }}$ varying according to Eq. 31); (b) - type (ii) initial conditions with fixed $\varepsilon^{(\mathrm{in})}=28.7\left[\mathrm{GeV} / \mathrm{fm}^{3}\right]$ (this is the value corresponding to the $q=1$ situation in (a)) and $\sigma$ varying according to Eq. (31).

(d)-(f)) and reproducing the initial energy $E_{\text {tot }}=26.1$ TeV. We start with $\varepsilon_{q}$ (panels (a) and (d) of Fig. 3) which is given by Eq. (29), use it to solve Eq. (16a) and find $T_{q}\left(\tau_{0}\right)$ (panels (b) and (e) of Fig. 3) and eventually obtain $s_{q}\left(\tau_{0}, \eta\right)$ using these results and Eq. (16c) (panels (c) and (f) of Fig. 3).

\section{Examples of $q$-hydrodynamical evolution of different thermodynamical quantities}

Let us now demonstrate examples of $q$-hydrodynamical evolution of different thermodynamical quantities and of the fluid rapidity. Calculations were performed using the method presented in Appendix B] In Fig 4 we present the evolution of the, respectively, energy density $\varepsilon_{q}$, temperature $T_{q}$ and entropy density $s_{q}$ using initial condi- 
TABLE I: List of parameters of the initial conditions used in Fig. 2. The initial temperature $T_{\text {in }} \equiv T\left(\tau_{0}, \eta=0\right)$ is shown for two types of EoS: for the relativistic nonextensive pion gas for some selected values of $q \geq 1$ and for the usual BG pion gas with $q=1$.

\begin{tabular}{|c|c|c|c|c|c|c|c|c|c|c|}
\hline \multicolumn{11}{|c|}{ Initial condition: $\sigma=1.25$ fixed } \\
\hline \multirow{2}{*}{$\begin{array}{c}E_{\text {tot }} \\
{[\mathrm{TeV}]}\end{array}$} & \multirow{2}{*}{$\begin{array}{c}\varepsilon^{(\mathrm{in})} \\
{\left[\mathrm{GeV} / \mathrm{fm}^{3}\right]}\end{array}$} & \multicolumn{4}{|c|}{$\sigma$} & \multirow[b]{2}{*}{ EoS } & \multicolumn{4}{|c|}{$T_{\text {in }}[\mathrm{GeV}]$} \\
\hline & & $q=1.00$ & $q=1.05$ & $q=1.10$ & $q=1.15$ & & $q=1.00$ & $q=1.05$ & $q=1.10$ & $q=1.15$ \\
\hline \multirow[b]{2}{*}{26.1} & \multirow[b]{2}{*}{27.8} & & 1.20 & 1.15 & 1.08 & nonex. $\pi$ gas & & 0.648 & 0.591 & 0.531 \\
\hline & & \multicolumn{4}{|c|}{1.25} & BG $\pi$ gas & \multicolumn{4}{|c|}{0.702} \\
\hline \multicolumn{11}{|c|}{ Initial condition: $\varepsilon^{(\mathrm{in})}=28.7 \mathrm{GeV} / \mathrm{fm}^{3}$ fixed } \\
\hline \multirow{2}{*}{$\begin{array}{c}E_{\text {tot }} \\
{[\mathrm{TeV}]}\end{array}$} & \multirow[t]{2}{*}{$\sigma$} & \multicolumn{3}{|c|}{$\varepsilon^{\text {(in) }}\left[\mathrm{GeV} / \mathrm{fm}^{3}\right.$} & & \multirow{2}{*}{ EoS } & \multicolumn{4}{|c|}{$T_{\mathrm{in}}[\mathrm{GeV}]$} \\
\hline & & $q=1.00$ & $q=1.05$ & $q=1.10$ & $q=1.15$ & & $q=1.00$ & $q=1.05$ & $q=1.10$ & $q=1.15$ \\
\hline \multirow[b]{2}{*}{26.1} & \multirow[b]{2}{*}{1.25} & & 25.9 & 22.4 & 16.8 & nonex. $\pi$ gas & & 0.631 & 0.556 & 0.464 \\
\hline & & \multicolumn{4}{|c|}{$\frac{1}{27.8}$} & $\mathrm{BG} \pi$ gas & \multicolumn{4}{|c|}{$\begin{array}{c}0.001+\frac{1}{10.000} \\
0.702\end{array}$} \\
\hline
\end{tabular}
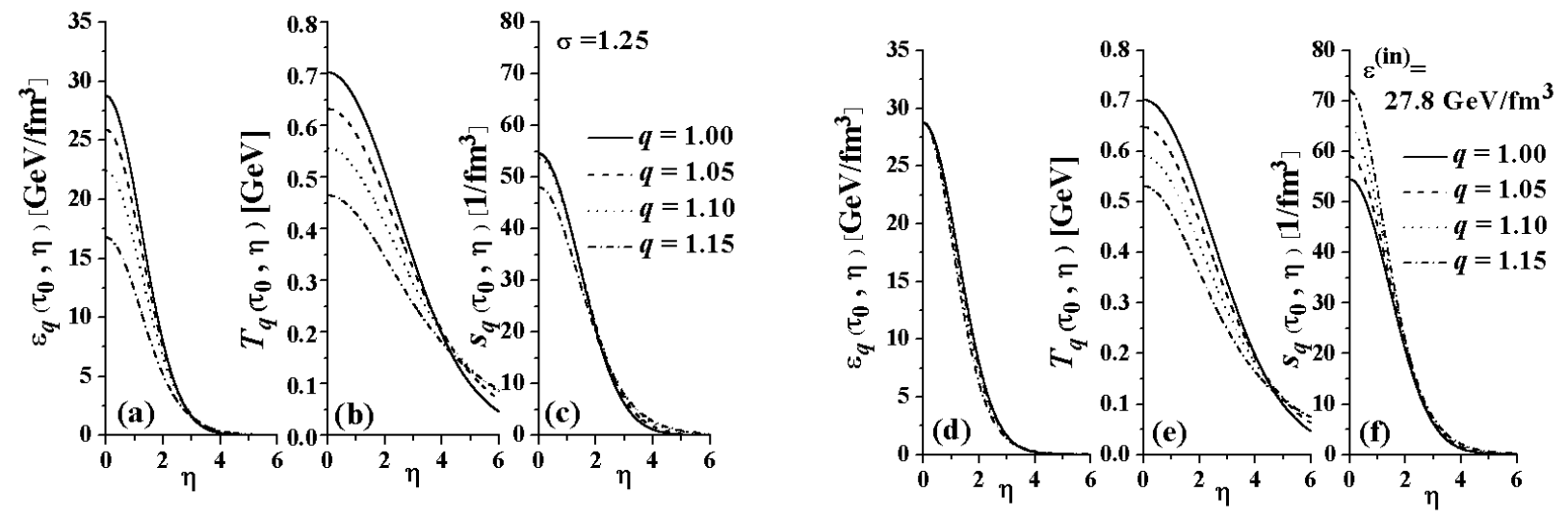

FIG. 3: Dependence of the initial conditions on the parameter $q$. The initial conditions for the energy density $\varepsilon_{q}\left(\tau_{0}, \eta\right)$ (panels (a) and (d)), the corresponding temperature $T_{q}\left(\tau_{0}, \eta\right)$ (panels (b) and (e)) and the entropy density $s_{q}\left(\tau_{0}, \eta\right)($ panels $(\mathrm{c})$ and $(\mathrm{f})$ ) are plotted for different values of $q$ and for two types of initial conditions: $(i)$ when the initial width $\sigma=1.25$ remains fixed (panels (a)-(c)) and (ii) when the initial maximal energy density $\varepsilon^{(\mathrm{in})}=27.8 \mathrm{GeV} / \mathrm{fm}^{3}$ remains fixed (panels (d)-(f)). In both cases we assume that $E_{\text {tot }}$ is the same for all $q$ 's and equal to $E_{\text {tot }}=26.1 \mathrm{TeV}$ whereas $\tau_{0}$ in Eq .(29) is put equal to $\tau_{0}=1.0$ $\mathrm{fm}$. Temperatures $T_{q}$ for different values of $q$ displayed on panels (b) and (e) are determined by solving Eq. (16a) whereas the entropy densities $s_{q}$ displayed on panels (c) and (f) are obtained from Eq. (16c) using values of $T_{q}$ displayed in panels (b) and (e).

tions discussed in Section IIIC (the exact values of relevant parameters for both types of initial conditions are listed in Table II). One can see that the initial functional forms of $\varepsilon_{q}(\tau, \eta), T_{q}(\tau, \eta)$ and $s_{q}(\tau, \eta)$ generally follow their original Gaussian shapes assumed at the initial time $\tau_{0}$ for $q=1.0,1.05$ and 1.1. On the other hand, during the whole hydrodynamical evolution both the energy density $\varepsilon_{q}$ and the temperature $T_{q}$ calculated for $q>1$ are smaller than those for $q=1$ for $\tau>\tau_{0}=1$ $\mathrm{fm}$ (see Table II). That is even true for the initial condition with fixed $\sigma=1.25$, for which the initial energy density $\varepsilon_{q=1.1}\left(\tau_{0}, \eta=3\right)>\varepsilon_{q=1.0}\left(\tau_{0}, \eta=3\right)$, in which case, after the $q$-hydrodynamical evolution is completed, one observes that $\varepsilon_{q=1}>\varepsilon_{q>1}$. The same trend is also observed for the temperature, i.e., $T_{q=1}>T_{q>1}$ for all $\tau$ and $\eta$. However, the corresponding entropy density $s_{q}$ evolves differently: for both type of initial conditions and any $\eta$, inequality relations between $s_{q=1.1}(\tau, \eta)$ and $s_{q=1.0}(\tau, \eta)$ given at initial $\tau=\tau_{0}$ are preserved during hydrodynamical evolution.

In what concerns the fluid rapidity $\alpha_{q}$, it is always set to be equal to $\alpha_{q}\left(\tau_{0}, \eta\right) \equiv \eta$ at $\tau=\tau_{0}$. However, the pressure gradient, which is characteristic to Gaussian type initial conditions applied here, accelerates the fluid, therefore $\alpha_{q}$ evolves with time $\tau$ (actually, this is true even for $q=1$ ), see Fig. 5. In these figures the fluid rapidity $\alpha_{q}$ (actually its deviation from the rapidity $\eta, \alpha_{q}-\eta$ ) is shown as a function of $\tau$ and the corresponding energy density $\varepsilon_{q}$. Notice that $\alpha_{q}-\eta \equiv 0$ at $\tau_{0}$ for the whole $\eta$ space (i.e. for all region of the $\varepsilon_{q}$ ). As shown in Fig. 5 , 
TABLE II: The values of energy density $\varepsilon_{q}$, entropy density $s_{q}$ and temperature $T_{q}$ at $\eta=0.0$ and $\eta=3.0$ and at $\tau=5.0$ and $25.0 \mathrm{fm}$ for $q=1.0$ and $q=1.1$ for two extremal types of initial conditions: with fixed $\sigma=1.25$ (upper panel) and with fixed $\varepsilon^{i n}=27.8 \mathrm{GeV} / \mathrm{fm}^{3}$ (lower panel).

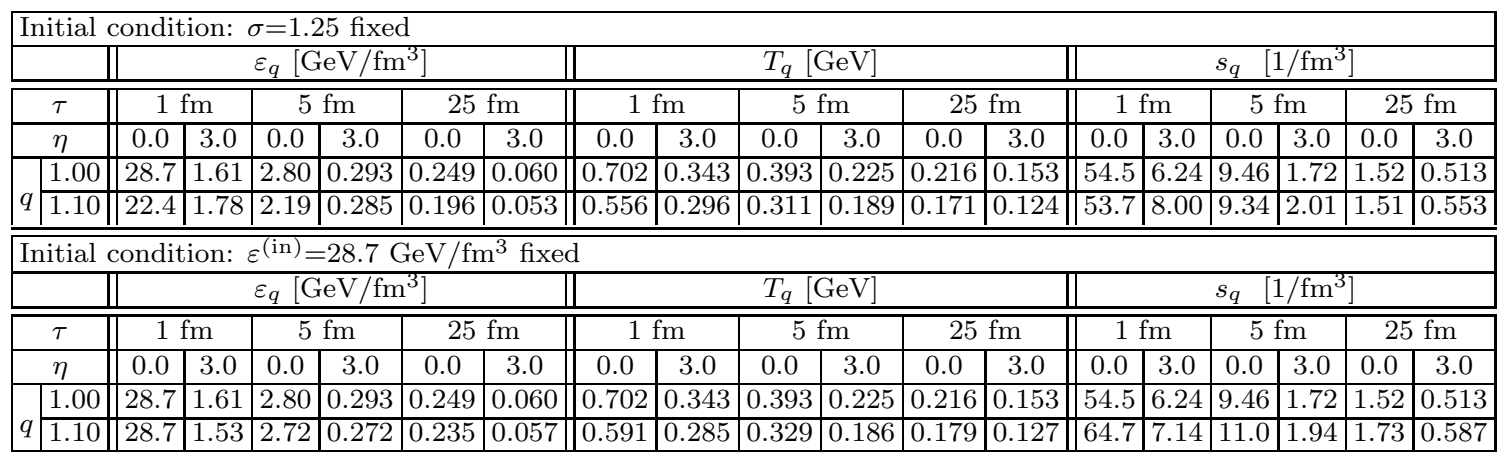
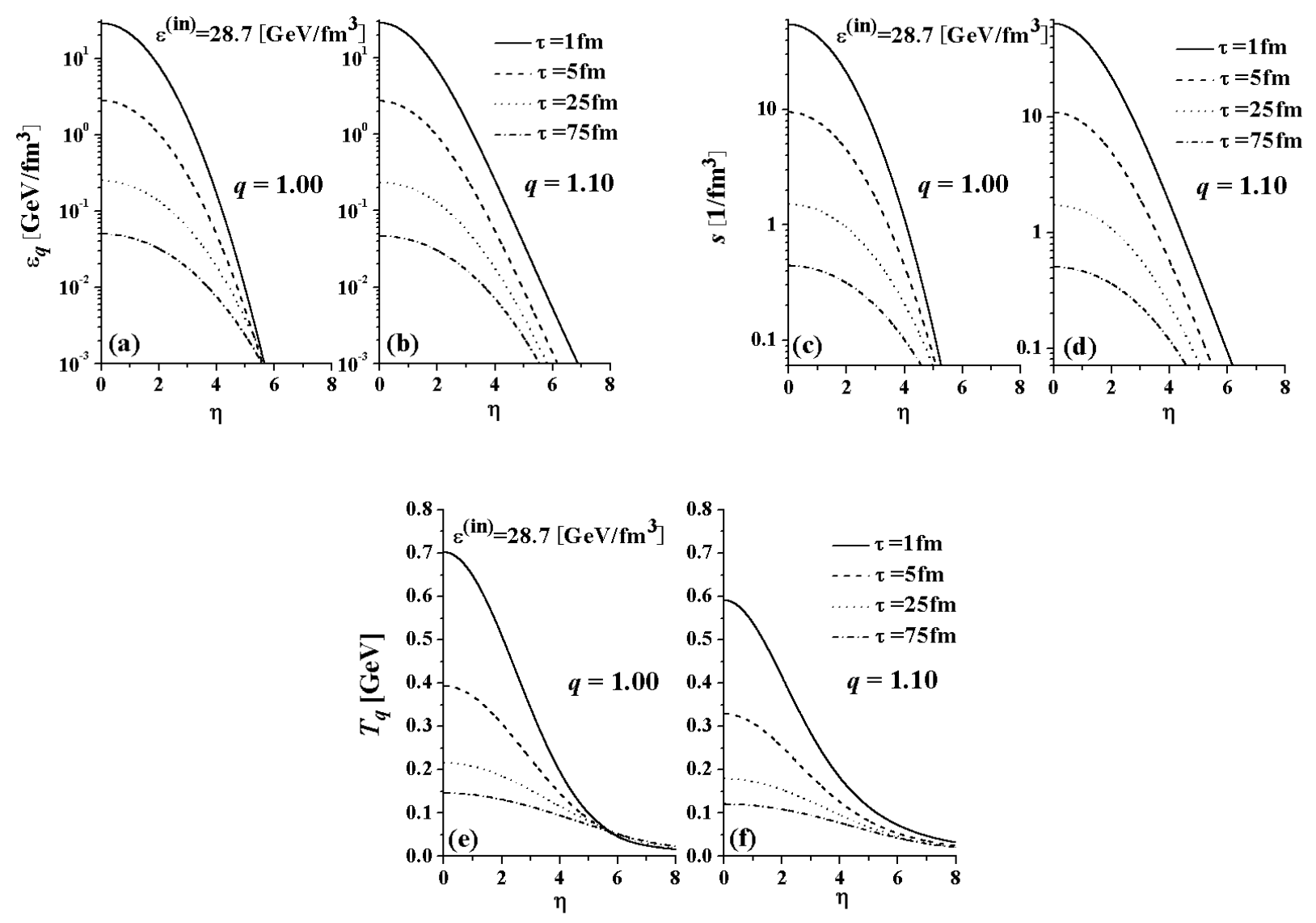

FIG. 4: Profiles of the energy density $\varepsilon_{q}$ (panels (a) and (b)), entropy density $s_{q}$ (panels (c) and (d)) and temperature $T_{q}$ (panels (e) and (f)) as functions of $\eta$ calculated for different proper times $\tau$ and different nonextensivity parameters $q$ by using initial conditions with fixed $\varepsilon^{(\mathrm{in})}=28.7 \mathrm{GeV} / \mathrm{fm}^{3}$. We also obtain similar results for $\varepsilon_{q}, T_{q}$ and $s_{q}$ with $\sigma=1.25$ fixed initial condition case.

the fluid rapidity $\alpha_{q}$ grows during the hydrodynamical expansion from its initial value $\alpha\left(\tau_{0}, \eta\right)=\eta$. One can observe that $\alpha_{q}$ for $q>1$ is decelerated compared to the usual hydrodynamic expansion (i.e., $\alpha_{q=1}>\alpha_{q>1}$ ).

To summarize this part: one observes that nonextensive fluid ( $q$-fluid with $q>1$ ) evolves more slowly than 
the ideal fluid (with $q=1$ ).

\section{E. Freezeout surface and single particle spectra}

We now present examples of single particle spectra emerging from our approach. We shall follow the simplest possibility in which they are expressed as an integral of the phase space particle density over a freeze-out surface $\Sigma_{\mathrm{f}}$ [47],

$$
\begin{aligned}
E \frac{d^{3} N}{d p^{3}} & =\frac{d^{3} N}{m_{\mathrm{T}} d m_{\mathrm{T}} d y d \phi} \\
& =\frac{g}{(2 \pi)^{3}} \int_{\Sigma_{\mathrm{f}}} d \sigma_{\mu}(x) p^{\mu} f_{\mathrm{eq}}(x, p) .
\end{aligned}
$$

In the $\tau-\eta$ metric the surface element of $\Sigma_{\mathrm{f}}$ given by

$$
d \sigma_{\mu}=\left(d \sigma_{\tau}, d \sigma_{\eta}\right)=A_{\mathrm{T}} \tau d \eta\left(1,-\frac{n_{\eta}}{n_{\tau}}\right)
$$

where $n_{\mu}$ is the normal covariant vector of the isothermals,

$$
n_{\mu}=\left(n_{\tau}, n_{\eta}\right)=\left(-\frac{\partial T}{\partial \tau},-\frac{\partial T}{\partial \eta}\right)
$$

and $A_{\mathrm{T}}$ is the transverse area of the generated fluid. In all examples of applications to $\mathrm{Au}+\mathrm{Au}$ collisions discussed in this paper we use $A_{\mathrm{T}}=6.5 \mathrm{fm}$. The momentum of the produced particle in the $\tau-\eta$ metric is given by

$$
p^{\mu}=\left[m_{\mathrm{T}} \cosh (y-\eta), \frac{1}{\tau} m_{\mathrm{T}} \sinh (y-\eta)\right]
$$

where $y$ is the observed rapidity (after freezeout). Using these expressions, the single particle density is given by:

$$
E \frac{d^{3} N}{d p^{3}}=\frac{d^{3} N}{m_{\mathrm{T}} d m_{\mathrm{T}} d y}=\frac{g A_{\mathrm{T}}^{2}}{4 \pi} \int d \eta \tau_{\mathrm{f}}(\eta)\left[m_{\mathrm{T}} \cosh (y-\eta)-\frac{1}{\tau} \frac{n_{\eta}(\eta)}{n_{\tau}(\eta)} m_{\mathrm{T}} \sinh (y-\eta)\right] f_{q}(y, \eta),
$$

where

$$
f_{q}(y, \eta)=\left[1-(1-q) \frac{m_{\mathrm{T}} \cosh (y-\eta)}{T_{\mathrm{F}}}\right]^{\frac{1}{1-q}}
$$

and $T_{\mathrm{F}}$ is the freezeout temperature which is given by the corresponding freezeout energy density $\varepsilon_{\mathrm{F}}$. In principle, the freezeout surface can be defined either as the surface of constant temperature $T_{\mathrm{F}}$, or as the surface of constant energy density $\varepsilon_{\mathrm{F}}$, or, finally, as the surface of constant entropy density $s_{\mathrm{F}}$ (cf. Table III). They all coincide in the usual extensive case $(q=1)$. In Fig. 6] we show as example freezeout surfaces (calculated for different values of parameter $q$ and for different initial conditions) for $T_{\mathrm{F}}=100 \mathrm{MeV}$. One observes a quite strong $q$ dependence of the freezeout surface characteristics on these parameters. These dependencies are much weaker when calculated for surface of constant energy density and even weaker for constant entropy density (not shown here explicitly). Note that values of $T_{\mathrm{F}}$ corresponding to freezeout conditions set by fixing $\varepsilon_{\mathrm{F}}$ or $s_{\mathrm{F}}$ now depend on the parameter $q$ (see Table III).

In Fig. 7 we show examples of single particle rapidity and transverse momentum spectra calculated for both types of initial conditions using $T_{\mathrm{F}}=100 \mathrm{MeV}$. Note that different types of the freezeout surface used are connected with using different sets of parameter $\left(q, T_{\mathrm{F}}\right)$, cf. Table III). Both distributions are sensitive to $q$, however, in the case of $d N / d y$ this dependence is almost entirely due to the $q$ dependence of the initial entropy density in

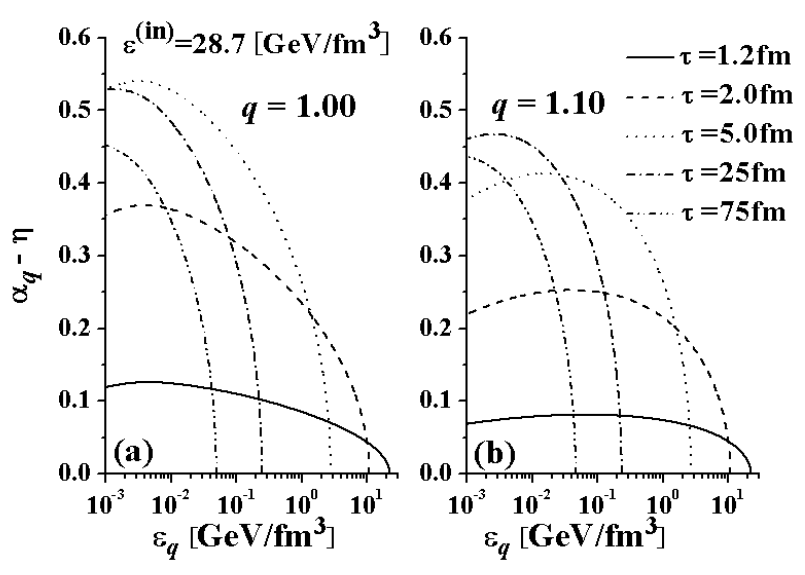

FIG. 5: (a) - evolution of the fluid rapidity $\alpha_{q}$ (presented as $\left.\alpha_{q}-\eta\right)$ as a function of the energy density $\varepsilon_{q}$ for different values of $\tau$ and for different nonextensivity parameters $q$ with fixed $\varepsilon^{(\mathrm{in})}=28.7 \mathrm{GeV} / \mathrm{fm}^{3}$. (b) - similar results for $\alpha_{q}-\eta$ using $\sigma=1.25$ fixed initial condition case.

the central region observed in Fig. 3 (panels (c) and (f)) and practically vanishes in the case of normalized distributions calculated for the constant $\varepsilon_{\mathrm{F}}$ freezeout surface, see the panels of Fig. 8 (a) and (b). This is because of the observed $q$ dependence of the corresponding to- 
TABLE III: The values of the freezeout temperatures $T_{\mathrm{F}}$ (in $\mathrm{MeV}$ ) for different freezeout (F.O.) conditions used and different values of $q$ investigated.

\begin{tabular}{|c||c|c|c|c|}
\hline$q$ & 1.00 & 1.05 & 1.10 & 1.15 \\
\hline \hline F.O. $=T_{\mathrm{F}}$ fixing & 100 & 100 & 100 & 100 \\
\hline F.O. $=\varepsilon_{\mathrm{F}}$ fixing & 100 & 91.8 & 83.2 & 74.3 \\
\hline F.O. $=s_{\mathrm{F}}$ fixing & 100 & 89.3 & 78.5 & 67.4 \\
\hline
\end{tabular}
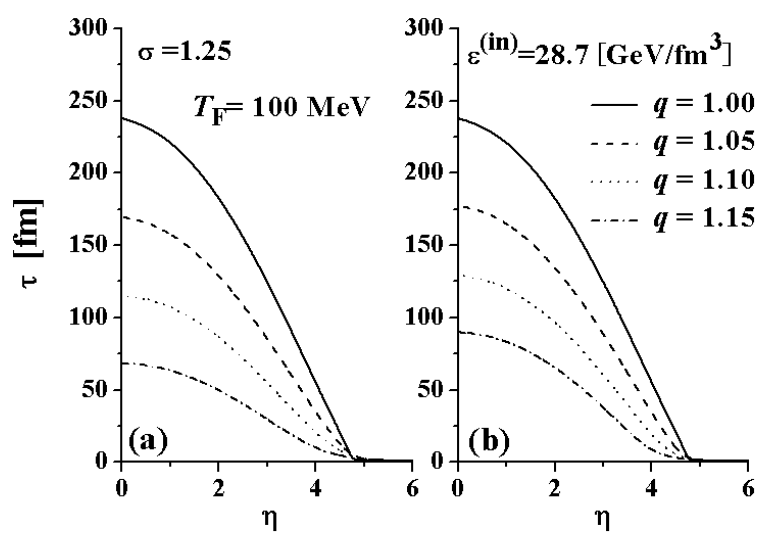

FIG. 6: Examples of freezeout surfaces of constant temperature $T_{\mathrm{F}}=100 \mathrm{MeV}$ calculated for different values of parameter $q$ and for different initial conditions: with constant $\sigma=1.25$ (a) and with constant energy density $\varepsilon_{\mathrm{F}}=9.82 \times 10^{-3} \mathrm{GeV} / \mathrm{fm}^{3}$ (b).

tal multiplicities and is connected with the increase of the entropy observed in nonextensive processes, see the panel Fig. 8 (c). We shall discuss this point in more detail below in Section $\mathrm{V}$. The weak residual $q$ dependence observed in this case can be attributed to the (apparently very weak) effects of the EoS and freezeout surface. In what concerns $p_{\mathrm{T}}$-spectra shown there for different initial conditions and freezeout surfaces, one observes a very strong dependence on $q$, which changes the slope of $p_{\mathrm{T}}$ considerably. It is interesting to note that, as seen in Fig. 7, the $p_{\mathrm{T}}$ distributions apparently are not sensitive neither to the type of initial conditions nor to the freezeout surfaces used.

In the $p_{\mathrm{T}}$ distributions, the slope depends on both $q$ and $T_{\mathrm{F}}$ and increasing $T_{\mathrm{F}}$ while keeping constant $q$ gives a similar effect as increasing $q$ at fixed $T_{\mathrm{F}}$. On the whole one observes tendency that transverse expansion as measured by these distributions gets stronger with increasing nonextensivity, i.e., with increasing $q$.
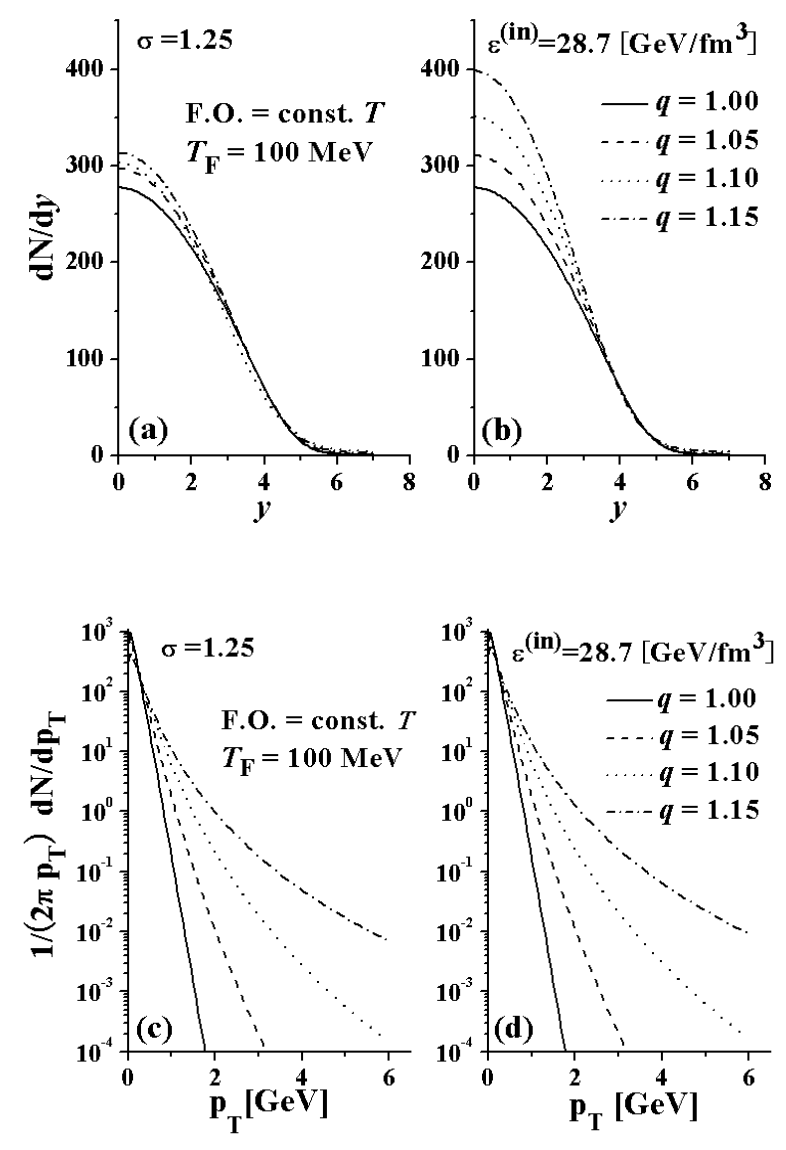

FIG. 7: $d N / d y$ and $p_{\mathrm{T}}$ spectra (panels (a)-(b) and (c)-(d), respectively) obtained from the $q$-hydrodynamical evolution with constant $T_{\mathrm{F}}=100 \mathrm{MeV}$ and for different values of parameter $q$ and for both types of initial conditions: with fixed $\sigma=1.25$ (panels (a) and (c)) and for fixed $\varepsilon^{(\mathrm{in})}=27.8$ $\mathrm{GeV} / \mathrm{fm}^{3}$ (panels (b) and (d) ). Rapidity spectra are obtained by integrating Eq. (37) over $p_{\mathrm{T}} \in(0,6.0) \mathrm{GeV} / \mathrm{c}$ whereas $p_{\mathrm{T}}$ spectra are obtained by integrating Eq.(37) over $|y| \leq 0.5$.

\section{COMPARISON WITH EXPERIMENTAL DATA}

We shall confront now our approach with experimental data. Because of still explanatory character of our work we limit ourselves only to comparison with some selected rapidity and $p_{\mathrm{T}}$ distributions. At this stage no attempts 

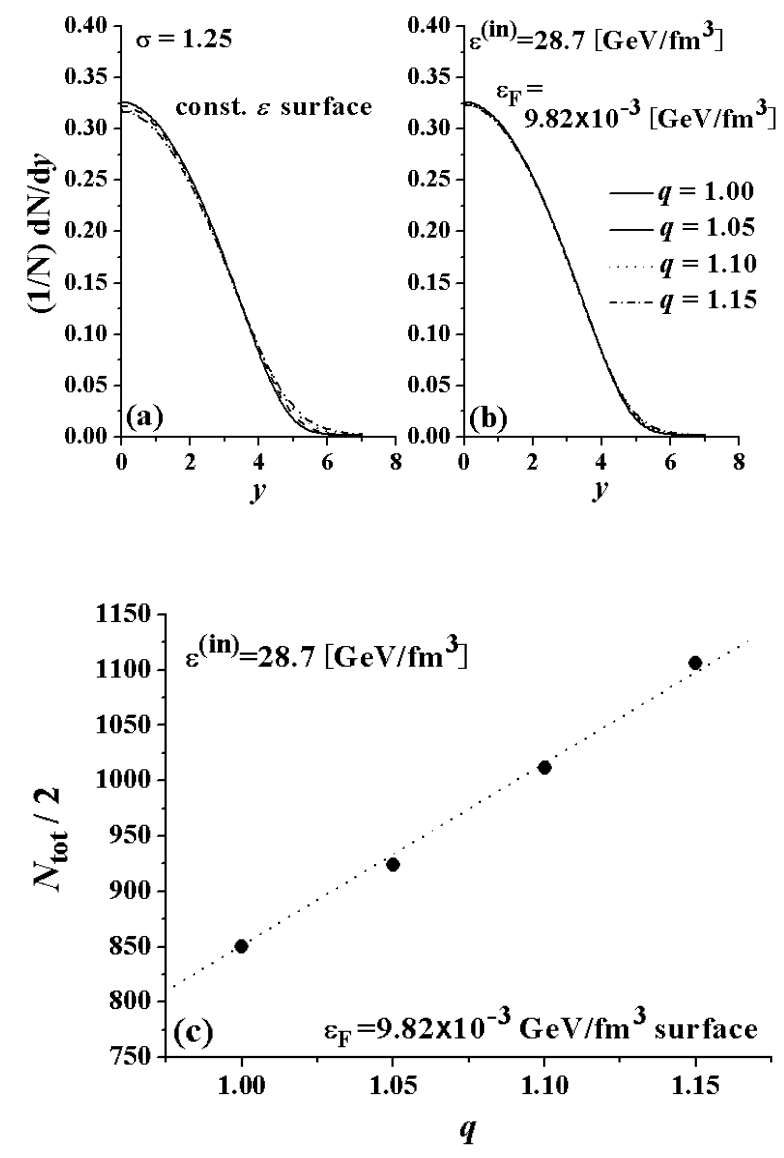

FIG. 8: (a) and (b) - normalized rapidity distributions $1 / N d N / d y$ (defined in the same way is in Fig. 7) calculated for constant energy density freezeout surface $\varepsilon_{\mathrm{F}}=9.82 \cdot 10^{-3}$ $\mathrm{GeV} / \mathrm{fm}^{3}$ for different values of $q$ using initial conditions with, respectively, fixed $\sigma=1.25$ and fixed $\varepsilon^{(\mathrm{in})}=28.7 \mathrm{GeV} / \mathrm{fm}^{3}$. Notice that there is only very weak $q$ dependence confined to small and large regions of rapidity $y$. (c) - $q$ dependence of the total multiplicity $N_{\text {tot }}$ obtained from the $q$-hydrodynamical evolution with fixed $\varepsilon^{(\mathrm{in})}=28.7 \mathrm{GeV} / \mathrm{fm}^{3}$ initial condition and $\varepsilon_{\mathrm{F}}=9.82 \cdot 10^{-3} \mathrm{GeV} / \mathrm{fm}^{3}$ freezeout condition. The total multiplicity $N_{\text {tot }}$ increases linearly with $q$.

for exact fits have been made. They must wait for a more detailed version which, for example, would account for the possible changes of the nonextensivity parameter $q$ during the collision process as mentioned in Section I. The same remarks apply to the potentially promising analysis of anisotropic flow or particle interferometry (for example, in the way as it was done in [33, 34, 35]), which we postpone until $1+2$ dimensional version of our approach accounting for expansion in transverse directions will be available in the future). Because, as was shown in Section IIIE the most sensitive for $q$ dependence are $p_{\mathrm{T}}$ distributions, we start with them and show in Fig. 9 that data by [48] prefer $q=1.08$ and $T_{\mathrm{F}}=100 \mathrm{MeV}$ (we attribute the visible discrepancy at largest values of $p_{\mathrm{T}}$ to the contamination from quark jets which carry large momentum in the initial stage of nuclear collisions and which are not accounted for $q$-hydrodynamical model. With these values of $q$ and $T_{\mathrm{F}}$ data provided by [49] for $d N / d y$ distributions and by [48] for $p_{\mathrm{T}}$ where compared with predictions of different initial conditions characterized by $\varepsilon^{(\text {in) }}$, see Fig. 10. As one can see, the $q$-hydrodynamical

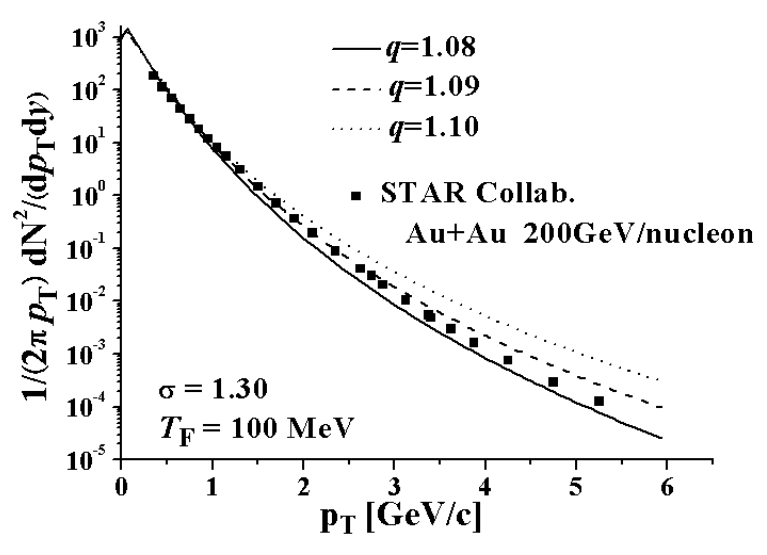

FIG. 9: Comparison of $q$-hydrodynamic model with experimental data observed by STAR collaboration [48] performed using $\sigma=1.30$ and $T_{\mathrm{F}}=100 \mathrm{MeV}$ for $q=1.08,1.09$ and 1.1 values (with corresponding values of $\varepsilon^{(\text {in) }}=21.2,205$ and 19.7 $\left.\mathrm{GeV} / \mathrm{fm}^{3}\right)$. The best agreement is obtained for $q=1.08$.

model with $q$-Gaussian initial condition can reproduce reasonably well both the rapidity and transverse momentum distribution data simultaneously. It should be stressed at this point that with the parameter $q>1$, which according to the general philosophy of the nonextensive approach accounts for all possible intrinsic fluctuations in the system [2, 6, 9], our model also accounts for the possible presence of resonances [12, 15] which therefore, to avoid double counting, should not be added independently. It must be noticed that in the present version we do not, in fact, account for the possible creation of a quark-gluon plasma (QGP) phase. For this one should use a more elaborate version of EoS than discussed here in Section IIIB. Notwithstanding all that, one can say that a simple $q$-hydrodynamical model reproduces experimental data reasonably well using $\varepsilon^{(\text {in })}=19.0 \div 22.3$ $\mathrm{GeV} / \mathrm{fm}^{3}(\sigma=1.28 \div 1.32), T_{\mathrm{F}}=100 \div 120 \mathrm{MeV}$, and $q=1.07 \div 1.08$. 


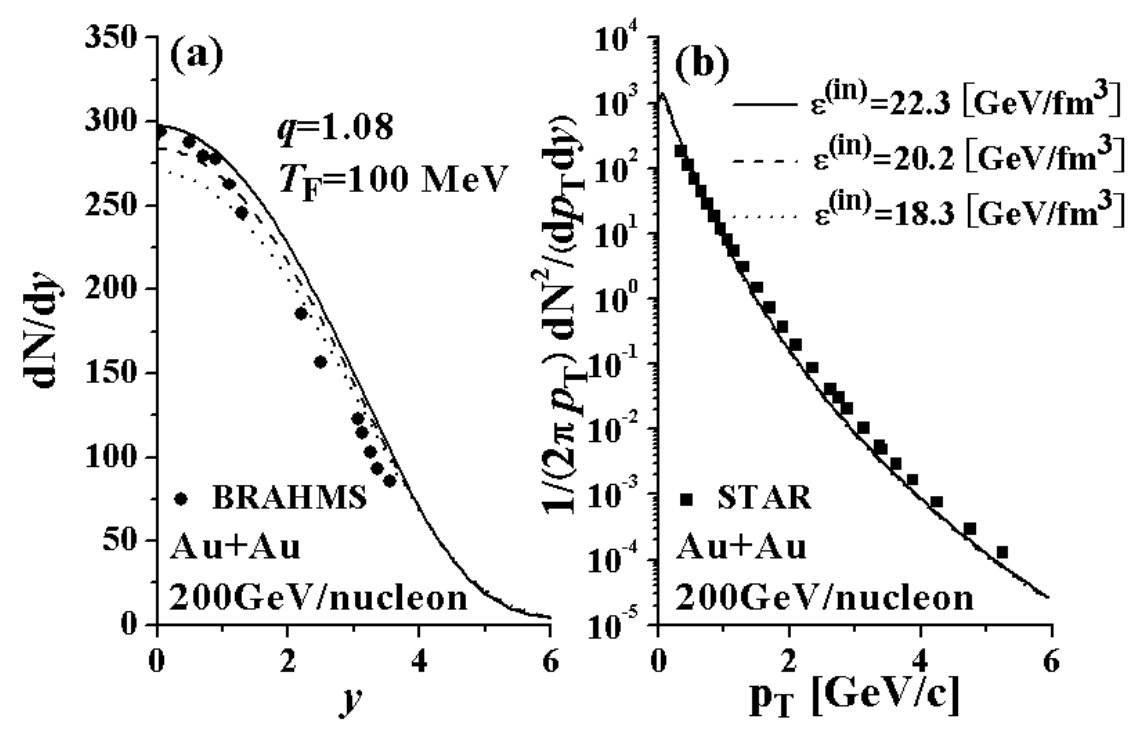

FIG. 10: Comparison of the $q$-hydrodynamic model with experimental data on rapidity [49] and $p_{\mathrm{T}}$ [48] distributions calculated for $q=1.08$ (fixed) and $T_{\mathrm{F}}=100 \mathrm{MeV}$, as in Fig. 9 but for different (Gaussian) initial conditions parametrized by $\varepsilon^{\text {(in) }}$.

\section{DISCUSSION: CAN PERFECT $q$-HYDRODYNAMICS MIMIC $d$-HYDRODYNAMICS?}

\section{A. Nonextensive/dissipative correspondence - formulation}

Our starting point is observation made at the end of Section IIID that $q$-fluid evolves more slowly than an ideal fluid. To this one can add observation from Section IIIE above that transverse expansion measured by the behavior of $p_{\mathrm{T}}$-spectra is much stronger in $q$-fluid. Those are precisely features observed in viscous fluids (cf., for example, 35]). Let us then treat these observation seriously and look more closely for the possible connections between $q$-fluid and viscous fluid apparently emerging from our $q$-hydrodynamical model.

Let us start with reminding the possible physical meaning of perfect $q$-hydrodynamics. It originated from the modified Boltzmann kinetic equation (3a) in which a new, $q$-generalized, version of the Boltzmann molecular chaos hypothesis [6, 7, 8, 9, 17, 37] has been used in the form of Eq. (4). It can be introduced in different ways 66 but effectively it always amounts to postulating a new kind of equilibrium, which includes some interactions and in which some stationary state is formed [8] summarily characterized by parameter $q$. In our case it leads to Eq. (20), which is formally identical to perfect hydrodynamical equation but with all usual ingredients replaced by their $q$-counterparts (perfect means here that there is nothing on the r.h.s. of Eq. (201)). It is natural to ask how Eq. (20) would look like when written in terms of the usual perfect hydrodynamic (with $q=1$ ) and some reminder depending on the parameter $q$. Because, as we have seen, in general $q$ differs only slightly from unity, $q-1 \ll 1$, it is tempting to simply expand Eq. (20) in small parameter $|q-1|[11,50]$. However, as shown in Appendix C, in such case one faces some unsurmountable problems because terms multiplying $|q-1|$ are not small enough in the whole of phase space. We shall therefore follow more general approach.

All our results presented above come from the Eq. (20), which is equation for perfect $q$-hydrodynamics. Notice that nonextensivity affects not only the thermodynamical quantities like energy density $\varepsilon$ and pressure $P$ but also the flow velocity field $u^{\mu}(x)$ :

$$
\begin{aligned}
\varepsilon(T) & \rightarrow \varepsilon_{q}\left(T_{q}\right) \equiv \varepsilon\left(T_{q}\right)+\Delta \varepsilon_{q}\left(T_{q}\right), \\
P(T) & \rightarrow P_{q}\left(T_{q}\right) \equiv P\left(T_{q}\right)+\Delta P_{q}\left(T_{q}\right), \\
u^{\mu}(x) & \rightarrow u_{q}^{\mu}(x) \equiv u^{\mu}(x)+\delta u_{q}^{\mu}(x) .
\end{aligned}
$$

where $u^{\mu}(x)$ is formally a solution of the equation which has form of dissipative hydrodynamical equation 23, 24, 25, 26, 27, 28, 29, 30, 31, 32],

$$
\left[\tilde{\varepsilon} u^{\mu} u^{\nu}-\tilde{P} \Delta^{\mu \nu}+2 W^{(\mu} u^{\nu)}+\pi^{\mu \nu}\right]_{; \mu}=0
$$


The notation used is:

$$
\begin{aligned}
& \tilde{\varepsilon}=\varepsilon_{q}+3 \Pi, \quad \tilde{P}=P_{q}+\Pi, \\
& W^{\mu}=w_{q}[1+\gamma] \Delta_{\lambda}^{\mu} \delta u_{q}^{\lambda}, \\
& \pi^{\mu \nu}=\frac{W^{\mu} W^{\nu}}{w_{q}[1+\gamma]^{2}}+\Pi \Delta^{\mu \nu} \\
& =w_{q} \delta u_{q}^{<\mu} \delta u_{q}^{\nu>}
\end{aligned}
$$

where $\tilde{\varepsilon}$ is energy density, $\tilde{P}$ pressure, $W^{\mu}$ energy or heat flow vector, $\pi^{\mu \nu}$ the (symmetric and traceless) shear pressure tensor and where

$$
\begin{aligned}
w_{q} & \equiv \varepsilon_{q}+P_{q}, \\
\gamma & \equiv u_{\mu} \delta u_{q}^{\mu}=-\frac{1}{2} \delta u_{q \mu} \delta u_{q}^{\mu},
\end{aligned}
$$

and

$$
\begin{aligned}
\left.A^{(\mu} B^{\nu}\right) & \equiv \frac{1}{2}\left(A^{\mu} B^{\nu}+A^{\nu} B^{\mu}\right), \\
a^{<\mu} b^{\nu>} & \equiv\left[\frac{1}{2}\left(\Delta_{\lambda}^{\mu} \Delta_{\sigma}^{\nu}+\Delta_{\sigma}^{\mu} \Delta_{\lambda}^{\nu}\right)-\frac{1}{3} \Delta^{\mu \nu} \Delta_{\lambda \sigma}\right] a^{\lambda} b^{\sigma}
\end{aligned}
$$

whereas

$$
\Pi \equiv \frac{1}{3} w_{q}\left[\gamma^{2}+2 \gamma\right]
$$

This last quantity can be regarded as a bulk pressure to be used below.

Now comes crucial point of our argumentation. To proceed further we shall assume that there exists some temperature $T$ and velocity field $\delta u_{q}^{\mu}$ satisfying the following relations:

$$
\begin{aligned}
& P(T)=P_{q}\left(T_{q}\right) \\
& \varepsilon(T)=\varepsilon_{q}\left(T_{q}\right)+3 \Pi
\end{aligned}
$$

( $\varepsilon$ and $P$ are energy density and pressure defined in the usual Boltzmann-Gibbs statistics, i.e., for $q=1$ ). In this case one can transform equation (39) into the following equation,

$$
\left[\varepsilon(T) u^{\mu} u^{\nu}-(P(T)+\Pi) \Delta^{\mu \nu}+2 W^{(\mu} u^{\nu)}+\pi^{\mu \nu}\right]_{; \mu}=0,
$$

which has the familiar form of the usual $d$ hydrodynamical equation. This means that perfect $q$-hydrodynamics represented by Eq. (20) can be regarded as being formally equivalent to some form of $d$-hydrodynamics as represented by Eq. (45). We shall call this observation the NexDC correspondence (and, respectively, we shall call Eq. (44) with Eq. (40b) and (40c) the NexDC relations). This observation can be traced back to the fact of generic non-conservation of global entropy in nonextensive systems, cf. Eq. (21), visualized in Fig. 8 as increase of the multiplicity with increasing $q$.

\section{B. Nonextensive/dissipative correspondence - consequences}

We shall now present shortly the most specific immediate consequences of NexDC correspondence: the entropy production and estimations of the corresponding transport coefficients.

\section{Entropy production in q-hydrodynamics}

Let us start with observation that Eq. (43) and NexDC relations (44) lead following form of $q$-enthalpy,

$$
\varepsilon_{q}\left(T_{q}\right)+P_{q}\left(T_{q}\right)=\frac{\varepsilon(T)+P(T)}{[1+\gamma]^{2}},
$$

which can be also used in definition of $\gamma$ because $w \equiv$ $T s=\varepsilon+P$ and $1 /(\gamma+1)=\sqrt{1-3 \Pi / w}(s$ is the entropy density in the usual Boltzmann-Gibbs statistics). Notice that in NexDC one has that

$$
\begin{aligned}
& W^{\mu} W_{\mu}=-3 \Pi w, \\
& \pi^{\mu \nu} W_{\nu}=-2 \Pi W^{\mu}, \\
& \pi_{\mu \nu} \pi^{\mu \nu}=6 \Pi^{2} .
\end{aligned}
$$

Suppose now that we define a true equilibrium state as a state with $q=1$, i.e., with no residual correlations between fluid elements and no intrinsic fluctuations present, with energy momentum tensor

$$
\mathcal{T}_{\text {eq }}^{\mu \nu} \equiv \mathcal{T}^{\mu \nu}=\varepsilon(T) u^{\mu} u^{\nu}-P(T) \Delta^{\mu \nu}
$$

and with equilibrium distribution given by the usual Boltzmann distribution,

$$
f_{\mathrm{eq}}(x, p)=\exp \left[-\frac{p^{\mu} u_{\mu}(x)}{k_{\mathrm{B}} T(x)}\right] .
$$

In this case the state characterized by $f_{q}(x, p)$ given by Eq. (14) must be regarded as some stationary state existing near equilibrium. Therefore, because we expect that $|q-1|$ is small, we can define a near equilibrium state defined by the correlation function $h_{q}$ in Eq. (41) for which the energy momentum tensor is $\mathcal{T}_{q}^{\mu \nu} \equiv$ $\left(\varepsilon_{q}+P_{q}\right) u_{q}^{\mu} u_{q}^{\nu}-P_{q} g^{\mu \nu}$, c.f., Eq. (15). It means then that we can write

$$
\mathcal{T}_{q}^{\mu \nu}=\mathcal{T}_{\text {eq }}^{\mu \nu}+\delta \mathcal{T}^{\mu \nu}
$$

where

$$
\delta \mathcal{T}^{\mu \nu}=-\Pi \Delta^{\mu \nu}+W^{\mu} u^{\nu}+W^{\nu} u^{\mu}+\pi^{\mu \nu} .
$$

Using now Eq. (44) we obtain the relation

$$
\gamma=\sqrt{1+\delta \epsilon_{q}}-1
$$

where

$$
\delta \epsilon_{q} \equiv \frac{\varepsilon(T)-\varepsilon_{q}\left(T_{q}\right)}{\varepsilon_{q}\left(T_{q}\right)+P_{q}\left(T_{q}\right)},
$$


which connects the velocity field $u$ (solution of the dissipative hydrodynamics given by Eq. (450) with the velocity field $u_{q}$ (solution of the $q$-hydrodynamics given by Eq. (20)).

In the $(1+1)$ dimensional case discussed here, one can always parameterize these velocity fields by using the respective fluid rapidities $\alpha_{q}$ and $\alpha$, $u_{q}^{\mu}(x)=\left[\cosh \left(\alpha_{q}-\eta\right), \frac{1}{\tau} \sinh \left(\alpha_{q}-\eta\right)\right]$ and $u^{\mu}(x)=$ $\left[\cosh (\alpha-\eta), \frac{1}{\tau} \sinh (\alpha-\eta)\right]$. Because $\gamma=u_{\mu} \delta u_{q}^{\mu}=$ $\cosh \left(\alpha_{q}-\alpha\right)-1$ one has that

$$
\cosh \left(\alpha_{q}-\alpha\right)=\sqrt{1+\delta \epsilon_{q}}
$$

which provides us with a connection between $u$ and $u_{q}$. From Eq. (53), one obtains finally

$$
\alpha=\alpha_{q}-\log \left(\epsilon_{q}+\sqrt{1+\delta \epsilon_{q}}\right) .
$$

We abandon here another solution of Eq. (53), namely that $\alpha=\alpha_{q}+\log \left(\epsilon_{q}+\sqrt{1+\delta \epsilon_{q}}\right)$, because it leads to the entropy reduction, i.e., for it $\left[s u^{\mu}\right]_{; \mu}<0$, for $q>1$. Taking the covariant derivative of Eq. (50) and multiplying it by $u_{\nu}$ we obtain

$$
u_{\nu} \mathcal{T}_{q ; \mu}^{\mu \nu}=T\left[s u_{\mu}\right]_{; \mu}+u_{\nu} \delta \mathcal{T}_{; \mu}^{\mu \nu}=0 .
$$

Therefore, although in ideal $q$-hydrodynamics the $q$ entropy is conserved, i.e., $\left[s_{q} u_{q}^{\mu}\right]_{; \mu}=0$, we can rewrite it in the form corresponding to dissipative fluid with $e n$ tropy production,

$$
\left[s u^{\mu}\right]_{; \mu}=-\frac{u_{\nu}}{T} \delta \mathcal{T}_{; \mu}^{\mu \nu} .
$$

To illustrate this we show in Fig. 11 the expected entropy production as given by Eq. (56). Notice that $s u_{: \mu}^{\mu}>0$ for the large $\eta$ region at any $\tau$ (but especially for the early stage of the hydrodynamical evolution). It supports therefore a dissipative analogy of the $q$-hydrodynamics mentioned before and leads us to very interesting conclusion that equilibrium state which is generated in the high-energy heavy-ion collisions may in fact be the $q$ equilibrium state which can be regarded as some stationary state near the usual (i.e., $q=1$ ) equilibrium state and which contains also some dissipative phenomena.

\section{Calculation of transport coefficients from q-hydrodynamics}

There are different formulations of $d$-hydrodynamics 23, 24, 25, 26, 27, 28, 29, 30, 31, 32]. In what follows we shall choose for comparison only the $2 n d$ order theory of dissipative fluids (in particular as given by [24, 25]) leaving investigations of other approaches from nonextensive perspectives for future investigations. As is known, this theory does not violate causality (at least not violate the global causality over distant scale given by the relaxation time), on the other hand it contains now some dissipative
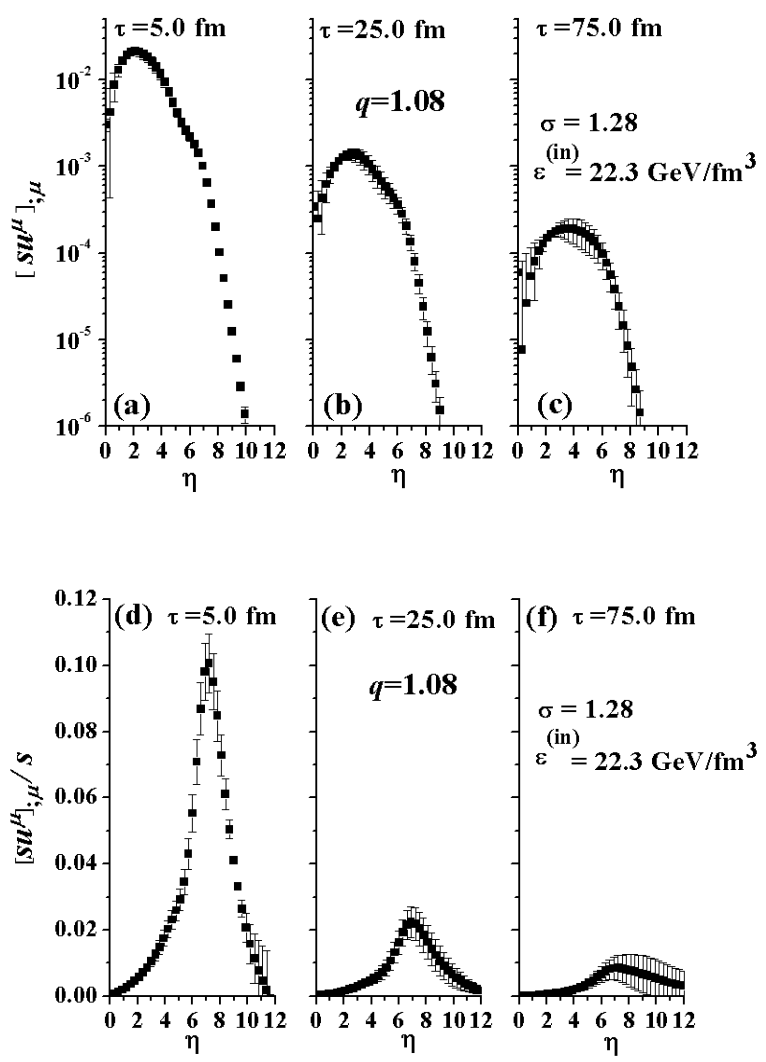

FIG. 11: Evolution of the entropy production $\left[s u^{\mu}\right]_{; \mu}$ (panels (a)-(c)) and ratio $\left[s u^{\mu}\right]_{; \mu} / s$ (panels (d)-(f)) as function of $\eta$ for different values of $\tau$ for $q=1.08$ with $\varepsilon^{\text {(in) }}=22.3 \mathrm{GeV} / \mathrm{fm}^{3}$ (or $\sigma=1.28$ ). The error bar is estimated by the value of $\left[s_{q} u_{q}^{\mu}\right]_{\mu}$ obtained in the numerical calculation which should be zero in an analytical calculation.

fluxes like heat conductivity, bulk and shear viscosity. We shall now see to what extent these transport coefficients can be calculated in $q$-hydrodynamics.

To this end let us start from considering more closely respective entropies. Dissipation is connected with the production of entropy and in [24, 25] the most general off-equilibrium four-entropy current $\sigma^{\mu}$ can be written as

$$
\sigma^{\mu}=P(T) \beta^{\mu}+\beta_{\nu}\left(\mathcal{T}_{\mathrm{eq}}^{\mu \nu}+\delta \mathcal{T}^{\mu \nu}\right)+Q^{\mu},
$$

where $\beta^{\mu} \equiv u^{\mu} / T$ and $Q^{\mu}=Q^{\mu}\left(\delta \mathcal{T}^{\mu \nu}\right)$ is some function which characterizes the off-equilibrium state. In the case of the $q$-entropy current (5) the NexDC conjecture (i.e., Eqs. (40b) and (46)) leads to the following off-equilibrium state:

$$
Q^{\mu}=Q_{\chi}^{\mu} \equiv \chi\left[s u^{\mu}+\frac{W^{\mu}}{T}\right]
$$


(with $\chi \equiv \frac{T}{T_{q}} \sqrt{1-\frac{3 \Pi}{w}}-1$ ) which results in

$$
\sigma_{q}^{\mu}\left(\equiv s_{q}^{\mu}\right)=s u^{\mu}+\frac{W^{\mu}}{T}+\chi\left\{s u^{\mu}+\frac{W^{\mu}}{T}\right\} .
$$

Notice that, because of the strict $q$-entropy conservation assumed here, using $Q^{\mu}=Q_{\chi}^{\mu}$ one always gets $\sigma_{q ; \mu}^{\mu}=$ 0 . It means that, although there is no production of $q$ entropy, there is production of the usual entropy, i.e., our $q$-system is really dissipative in the usual meaning of this word.

Let us now be more specific and use the most general algebraic form of $Q^{\mu}$, calculated up to the second order in the dissipative flux, as given by [25]

$$
\begin{gathered}
Q_{2 \mathrm{nd}}^{\mu}=\frac{\left[-\beta_{0} \Pi^{2}+\beta_{1} W_{\nu} W^{\nu}-\beta_{2} \pi_{\nu \lambda} \pi^{\nu \lambda}\right]}{2 T} u^{\mu} \\
-\frac{\alpha_{0} \Pi W^{\mu}}{T}+\frac{\alpha_{1} \pi^{\mu \nu} W_{\nu}}{T} .
\end{gathered}
$$

Here $\beta_{i=1,2,3}$ are the corresponding thermodynamic coefficients for the, respectively, scalar, vector and tensor dissipative contributions to the entropy current whereas $\alpha_{i=0,1}$ are the corresponding viscous/heat coupling coefficients. The $\Pi$ is bulk pressure defined before in Eq. (43) [67]. In the NexDC one has that

$$
Q_{2 \mathrm{nd}}^{\mu} \rightarrow \Gamma_{2 \mathrm{nd}} s u^{\mu}+\Upsilon_{1 \mathrm{st}} \frac{W^{\mu}}{T},
$$

where

$$
\begin{aligned}
& \Gamma_{2 \text { nd }} \equiv-\frac{3 \beta_{1}}{2} \Pi-\frac{\left(\beta_{0}+6 \beta_{2}\right)}{2 w} \Pi^{2}, \\
& \Upsilon_{1 \mathrm{st}} \equiv-\left(\alpha_{0}+2 \alpha_{1}\right) \Pi .
\end{aligned}
$$

$Q^{\mu}$ can be then expressed by polynomials in the bulk pressure $\Pi$ defined by Eq. (43). It is then natural to expect that the most general entropy current in the NexDC approach has form:

$$
Q_{\text {full }}^{\mu}=\Gamma(\Pi) s u^{\mu}+\Upsilon(\Pi) \frac{W^{\mu}}{T},
$$

where $\Gamma, \Upsilon$ are (in general infinite) series in powers of the bulk pressure $\Pi$. In this sense the $Q_{\text {full }}^{\mu}$ can be regarded as the full order dissipative current.

In general one has entropy production/reduction, $\sigma_{; \mu}^{\mu} \neq 0$, however in case when $\Gamma(\Pi)=\Upsilon(\Pi)=\chi$ one has $\sigma_{\chi ; \mu}^{\mu}=0$ so one can write the full order dissipative entropy current as

$$
Q_{\text {full }}^{\mu}=(\chi+\xi) s u^{\mu}+(\chi-\xi) \frac{W^{\mu}}{T},
$$

where $\Gamma$ and $\Upsilon$ are determined by $\chi \equiv(\Gamma+\Upsilon) / 2$ and $\xi \equiv(\Gamma-\Upsilon) / 2$. From two solution for $(\Gamma, \Upsilon)$,

or

$$
\frac{\Gamma}{2} \equiv \frac{T}{T_{q}}\left(\sqrt{1-\frac{3 \Pi}{w}}-1\right), \quad \frac{\Upsilon}{2} \equiv \frac{T-T_{q}}{T_{q}}
$$

$$
\frac{\Gamma}{2} \equiv \frac{T-T_{q}}{T_{q}}, \quad \frac{\Upsilon}{2} \equiv \frac{T}{T_{q}}\left(\sqrt{1-\frac{3 \Pi}{w}}-1\right),
$$

only (65a) is acceptable because only for it $u_{\mu} Q_{\text {full }}^{\mu} \leq 0$ (i.e., entropy is maximal in the equilibrium [25] and this is because $\left(T-T_{q}\right) / T_{q}$ is always positive for $\left.q \geq 1\right)$. In this way we finally arrive at the following possible expression for the full order dissipative entropy current in the NexDC approach:

$$
\begin{aligned}
& \sigma_{\text {full }}^{\mu} \equiv s u^{\mu}+\frac{W^{\mu}}{T}- \\
& \quad-\frac{2 T}{T_{q}}\left[1-\sqrt{1-\frac{3 \Pi}{w}}\right] s u^{\mu}+\frac{2\left(T-T_{q}\right)}{T_{q}} \frac{W^{\mu}}{T} .
\end{aligned}
$$

Limiting ourselves to situations when $T / T_{q} \approx 1$ and neglecting terms higher than $\mathcal{O}(3 \Pi / w)^{2}$, one obtains that

$$
Q_{\text {full }}^{\mu} \approx\left[-\left(\frac{3 \Pi}{w}\right)-\frac{1}{4}\left(\frac{3 \Pi}{w}\right)^{2}\right] s u^{\mu} .
$$

Comparing now Eqs. (62) and (67) one gets that 68]

$$
\beta_{1}=\frac{2}{w}, \quad \beta_{0}+6 \beta_{2}=\frac{9}{2 w}, \quad \alpha_{0}+2 \alpha_{1}=0 .
$$

Since in the Israel-Stewart theory [24] the relaxation time $\tau$ is proportional to thermodynamical coefficients $\beta_{0,1,2}$, it is naturally to assume that in our NexDC case $\tau \propto 1 / w$, i.e., it is proportional to the inverse of the enthalpy (notice that for classical Boltzmann gas of massless particles one obtains $\beta_{2}=3 / w[25,32]$ ).

We shall now derive the bulk and shear viscosities emerging from the NexDC approach. Let us start with observation that the local entropy production by the full order entropy current Eq. (66) can be also written as

$$
\sigma_{\text {full } ; \mu}^{\mu}=\left[(1+\chi) \Phi^{\mu}\right]_{; \mu}+\left[\xi \Psi^{\mu}\right]_{; \mu},
$$

where $\Phi^{\mu}=s u^{\mu}+\frac{W^{\mu}}{T}$ and $\Psi^{\mu}=s u^{\mu}-\frac{W^{\mu}}{T}$. Because conservation of $q$-entropy, $\sigma_{q ; \mu}^{\mu}=0$, is equivalent to $[(1+$ $\chi{ }^{\mu} \Phi_{; \mu}=0$, therefore using Eq. (46) one gets that

$$
\Psi^{\mu}=-\frac{W^{\nu} W_{\nu}}{3 \Pi T} u^{\mu}+\frac{W_{\nu}}{2 \Pi T} \pi^{\mu \nu}
$$

and (see Appendix Dfor details of derivation of Eqs. (71) and (73))

$$
\sigma_{\text {full } ; \mu}^{\mu}=-\frac{\Pi}{T}\left(w u^{\mu} X_{\mu}\right)-\frac{W^{\mu}}{T} \tilde{Y}_{\mu}+\frac{\pi^{\mu \nu}}{T} Z_{\mu \nu},
$$

where

$$
\begin{aligned}
X_{\mu} & =-\frac{\xi}{\Pi}\left[\frac{\partial_{\mu} \Pi}{\Pi}+\frac{\partial_{\mu} T}{T}+\frac{\partial_{\mu} \xi}{\xi}\right] \\
Y_{\mu} & =\frac{\xi}{\Pi}\left[\frac{2}{3} u^{\nu} W_{\mu ; \nu}+\frac{1}{3} W_{\mu} u_{; \nu}^{\nu}-\frac{1}{2} \pi_{\mu ; \nu}^{\nu}\right], \\
Z_{\mu \nu} & =\frac{\xi}{\Pi}\left[\frac{1}{2} W_{\nu ; \mu}\right]
\end{aligned}
$$

and

$$
\tilde{Y}_{\mu}=Y_{\mu}-\Pi X_{\mu}, \quad \tilde{Z}_{\mu \nu} \equiv Z_{\mu \nu}+\frac{\tilde{Y}_{\mu} W_{\nu}}{2 \Pi} .
$$


One can now use Eq. (47) to eliminate term proportional to heat flow $\frac{W^{\mu}}{T}$. In this way one avoids explicit contribution to entropy production from the heat flow $\frac{W^{\mu}}{T}$, which is present in Eq. (71) when one discuss baryon free fluid, in which case the necessity to use Landau frame would appear. As one can see Eq. (69) is covariant and therefore it does not depend on the frame used. After that one obtains that

$$
\sigma_{\text {full } ; \mu}^{\mu}=-\frac{\Pi}{T}\left(w u^{\mu} X_{\mu}\right)+\frac{\pi^{\mu \nu}}{T} \tilde{Z}_{\mu \nu} .
$$

Eq. (73) can be now used to find the bulk and shear viscosities from $\sigma_{\text {full; } \mu}^{\mu}$ given by Eq. (69). The positive transport coefficients, bulk viscosity $\zeta$ and shear viscosity $\eta$, can be estimated by writing the entropy production $\sigma_{\text {full; } \mu}^{\mu}$ in the following form:

$$
\sigma_{\text {full } ; \mu}^{\mu}=\frac{\Pi^{2}}{\zeta T}+\frac{\pi^{\mu \nu} \pi_{\mu \nu}}{2 \eta T} \geq 0
$$

and using Eq. (47). We arrive then at the sum rule connecting transport coefficients (expressed as ratios of bulk and shear viscosities over the entropy density $s$ ),

$$
\frac{1}{\zeta / s}+\frac{3}{\eta / s}=\frac{w \sigma_{\text {full } ; \mu}^{\mu}}{\Pi^{2}} .
$$

This is as far as we can go. The heat conductivity, as shown above, can be expressed by two other transport coefficients for which we have only one equation in the form of sum rule (75). To proceed any further and to disentangle (75) one has to add some additional input. Suppose then that we are interested in the extremal situation, when total entropy is generated by action of shear viscosity only. In this case one can rewrite Eq. (73) as

$$
\sigma_{\text {full } ; \mu}^{\mu}=\frac{\pi^{\mu \nu}}{T}\left[-\frac{\pi_{\mu \nu}}{6 \Pi}\left(w u^{\lambda} X_{\lambda}\right)+\tilde{Z}_{\mu \nu}\right]
$$

resulting in

$$
\frac{\eta}{s}=\frac{\gamma(\gamma+2)}{3(\gamma+1)^{2}}\left[\frac{\pi^{\mu \nu}}{\Pi} \frac{\tilde{Z}_{\mu \nu}}{T}-s u^{\lambda} X_{\lambda}\right]^{-1} .
$$

Note that Eq. (77) allows all values of $\eta / s$, in particular that $\eta / s<\frac{1}{4 \pi}$, what violates the limit obtained from AdS/CFT correspondence that $\eta / s \geq 1 / 4 \pi$ [36]. To impose this limit we shall now use Eq. (75). This can be done only in the region where r.h.s. of Eq. (77) is smaller than (or equal to) $1 / 4 \pi$ (in which case we put $\eta / s=1 / 4 \pi)$, otherwise, because of our earlier assumed limitation we put $\zeta / s=0$ and use Eq. (77) to evaluate $\eta / s$. The corresponding results for $\zeta / s$ and $\eta / s$ are shown in Fig 12 (a) and (b), respectively. Notice that when the r.h.s of Eq. (77) approaches $1 / 4 \pi, \zeta / s$ given by Eq. (75) approaches infinity. All curves presented in Fig. 12 were calculated for $\mathrm{Au}+\mathrm{Au}$ collisions with identical set of parameter as the best fit presented in Fig. 10 above.

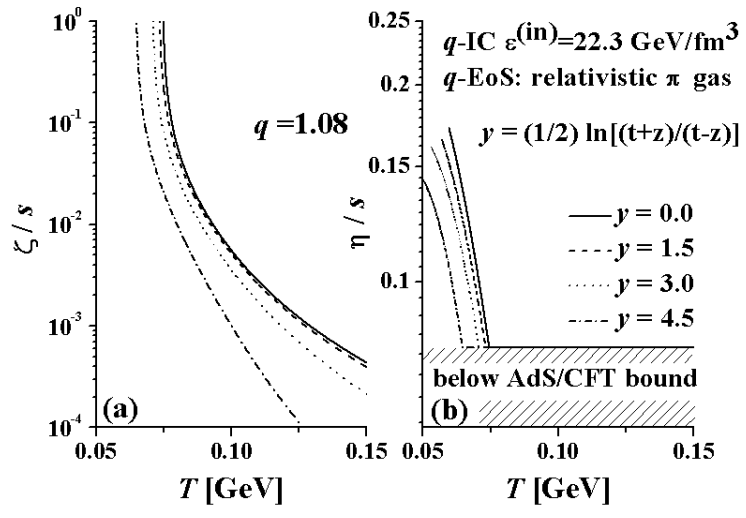

FIG. 12: The NexDC predictions for the ratios of bulk (panel (a)) and shear (panel (b)) viscosities over the entropy density, $\zeta / s$ and $\eta / s$, as function of temperature $T$ and calculated for a number of space-time rapidities $y \equiv \frac{1}{2} \ln \frac{t+z}{t-z}$ (the same as in Eq. (24b) using $q$-hydrodynamical model).

\section{SUMMARY AND CONCLUSIONS}

We have presented a nonextensive version of the hydrodynamical model for multiparticle production processes, the $q$-hydrodynamical model, which is based on the nonextensive statistics represented by Tsallis entropy and indexed by the nonextensivity parameter $q$. In doing so we have followed the usual approach originating in the appropriate kinetic equations formulated in nonextensive form in [17]. We have found the nonextensive entropy current which satisfies not only the nonextensive $H$-theorem, Eq. (5), but also the $q$-version of thermodynamical relations Eq. (17). The $(1+1)$ dimensional $q$-hydrodynamics with the $q$-Gaussian initial condition and the $q$-EoS can reasonably reproduce the single particle spectra observed at RHIC energy for $q=1.07 \div 1.08$ for $T_{\mathrm{F}}=100 \div 120 \mathrm{MeV}$ if quark jet contributions to $p_{\mathrm{T}}$ spectra are small, i.e., up to transverse momentum range around $p_{\mathrm{T}} \leq 6.0 \mathrm{GeV} / \mathrm{c}$. We also found a possible correspondence between the $q$-hydrodynamics and the usual $(q=1) d$-hydrodynamics (NexDC) as provided by the Eq. (45) with NexDC relations Eq. (44). Based on this correspondence, we have evaluated the entropy production in relativistic heavy-ion collisions at RHIC energy using results of our perfect $q$-hydrodynamics (understood as an approach without any $q$-viscosity effects added). The fact that when comparing with data one finds that $q>1$ means that one, indeed, has some dynamic factors present, detailed form of which is not yet disclosed but which summarily can be accounted for by the nonextensive approach and which action is summarized by the parameter $q-1$.

In what concerns the obtained $p_{\mathrm{T}}$ dependence, our formula continues attempts made to interpret power-law spectra as new kind of equilibrium phenomena for the 
whole $p_{\mathrm{T}}$ range pushing the usual interpretation via onset of "hard" collisions (imposed on the "soft" ones) to really high values of $p_{\mathrm{T}}$ (cf., [8, 11, 15] and references therein). In such approach there is no characteristic scale at which the transition from "soft" (or locally thermalized) to "hard" (or unthermalized) dynamics occurs which appears in the conventional descriptions using viscous hydrodynamics, as for example in [34, 51].

One of the results of our investigation is that fluctuations in the initial conditions seem to be the most important part of the hydrodynamical model, which by using Tsallis statistics, attempts to account for any possible fluctuations in some general, model independent, way. This is quite reasonable result because at the initial stage our system consists of a relatively small number of degrees of freedom and is therefore more sensitive to any fluctuations. On the contrary, at freezeout this number is much bigger and the system is only weakly responding to any fluctuations. This finding agrees nicely with recent analysis of the elliptical flow performed by using a hydrodynamical approach in which an attempt was made to account for fluctuations (without, however, using $q$-statistics), see [52]. On the other hand, however, it should be remembered that analysis presented here is considerably simplified by using the same nonextensivity parameter $q$ at all stages of the collision process. There is therefore room for improvements which will facilitate comparison with data. One can argue that intrinsic fluctuations existing in different stages of the collision process are of different (albeit connected) dynamical origin and therefore parameters $q$ for the initial conditions, for the EoS and, finally, for the hydrodynamical expansion should be allowed to have different values (and should be also different for the longitudinal and transverse dynamics). The other problem would be how to connect our $q$ parameter expressing fluctuations with fluctuations in all momentum observables as seen when analyzing a non-ideal liquid as it was done, for example, in [53]. We plan to address these problems elsewhere. In any case, similarly to the fact that concept of ideal fluid is never realized in nature [35] (the bound of $\eta / s \geq 1 / 4 \pi$ found in [36] being strong argument supporting this), the $q=1$ case should be replaced by investigations of the $q$-fluid with $q>1$.

In this context the natural question arises concerning the deeper physical meaning of the $q$-hydrodynamic proposed here. The most important observation dis- cussed in Section $\nabla$ is the apparent correspondence found between the perfect $q$-hydrodynamics and the usual $d$ hydrodynamics, which we call the NexDC correspondence. It allows calculation of transport coefficients of viscous fluid in terms of parameters of $q$-(ideal) fluid, i.e., essentially as dependent on single parameter which, as it was already stressed many times, represents summary effect of many possible dynamical factors, without entering into dynamical details (i.e., in a purely phenomenological way). The detail discussion of the NexDC phenomenon is, however, outside the scope of present paper and we plan to address is elsewhere.

We close by remark that hydrodynamics can also be derived using Information Theory with its method of maximization of information entropy under some specific constraints 54. It is therefore plausible that our results could also be derived using a nonextensive version of Information Theory (in the way as 13, 55] can be regarded as a nonextensive generalization of the Information Theory approach to single particle distributions obtained in the multiparticle production processes proposed in [56]). We shall not pursue this possibility here.

\section{Acknowledgments}

One of us (TO) thanks the Yukawa Institute for Theoretical Physics at Kyoto University. Discussions during the YITP workshop YITP-W-07-07 on "Thermal Quantum Field Theories and Their Applications" were useful to complete this work. Partial support (GW) of the Ministry of Science and Higher Education under contracts 1P03B02230 and CERN/88/2006 is acknowledged.

\section{APPENDIX A: DERIVATION OF EQS.(26) AND (27)}

Consider some details of the $(1+1)$ dimensional relativistic hydrodynamics under assumption that one ignores the transverse expansion of the fluid. With $g_{\mu \nu}=$ $\operatorname{diag}\left(1,-\tau^{2}\right)$ and four fluid velocity $u_{\mu}=\left[\cosh \left(\alpha_{q}-\right.\right.$ $\left.\eta),-\tau \sinh \left(\alpha_{q}-\eta\right)\right]$ the projection matrix is equal to:

$$
\Delta_{q \mu \nu} \equiv g_{\mu \nu}-u_{q \mu} u_{q \nu}=\left[\begin{array}{lc}
-\sinh ^{2}\left(\alpha_{q}-\eta\right) & \tau \cosh \left(\alpha_{q}-\eta\right) \sinh \left(\alpha_{q}-\eta\right) \\
\tau \cosh \left(\alpha_{q}-\eta\right) \sinh \left(\alpha_{q}-\eta\right) & -\tau^{2} \cosh ^{2}\left(\alpha_{q}-\eta\right)
\end{array}\right]
$$

The nonvanishing components of Christoffel symbols are $\Gamma_{\tau \eta}^{\eta}=\Gamma_{\eta \tau}^{\eta}=1 / \tau$ and $\Gamma_{\eta \eta}^{\tau}=\tau$, therefore covariant derivative of fluid velocity, which is defined by $u_{; \nu}^{\mu}=$ 
$\partial_{\nu} u_{\mu}+\Gamma_{\lambda \nu}^{\mu} u^{\lambda}$, has the following form:

$$
\begin{aligned}
& u_{q ; \tau}^{\tau}=\sinh \left(\alpha_{q}-\eta\right) \frac{\partial \alpha_{q}}{\partial \tau}, u_{q ; \tau}^{\eta}=\frac{1}{\tau} \cosh \left(\alpha_{q}-\eta\right) \frac{\partial \alpha_{q}}{\partial \tau} \\
& u_{q ; \eta}^{\tau}=\sinh \left(\alpha_{q}-\eta\right) \frac{\partial \alpha_{q}}{\partial \eta}, u_{q ; \eta}^{\eta}=\frac{1}{\tau} \cosh \left(\alpha_{q}-\eta\right) \frac{\partial \alpha_{q}}{\partial \eta} .
\end{aligned}
$$

Using these expressions, one obtains

$$
\begin{aligned}
u_{q}^{\mu} \Delta_{q \tau \nu} u_{q ; \mu}^{\nu} & =\cosh \left(\alpha_{q}-\eta\right) \sinh \left(\alpha_{q}-\eta\right) \frac{\partial \alpha_{q}}{\partial \tau} \\
& +\frac{1}{\tau} \sinh ^{2}\left(\alpha_{q}-\eta\right) \frac{\partial \alpha_{q}}{\partial \eta} \\
u_{q}^{\mu} \Delta_{q \eta \nu} u_{q ; \mu}^{\nu} & =-\tau \cosh ^{2}\left(\alpha_{q}-\eta\right) \frac{\partial \alpha_{q}}{\partial \tau} \\
& -\cosh \left(\alpha_{q}-\eta\right) \sinh \left(\alpha_{q}-\eta\right) \frac{\partial \alpha_{q}}{\partial \eta} .
\end{aligned}
$$

Because $g_{; \nu}^{\mu \nu}=0$ for $\mu, \nu=\tau$ and $\eta$, Eq. (23) is reduced to the following two equations:

$$
\begin{aligned}
\left(\varepsilon_{q}+P_{q}\right) & \left\{u_{q}^{\mu} \Delta_{q \tau \nu} u_{q ; \mu}^{\nu}\right\} \\
& -\Delta_{q \tau \tau} \frac{\partial P_{q}}{\partial \tau}+\frac{1}{\tau^{2}} \Delta_{q \tau \eta} \frac{\partial P_{q}}{\partial \eta}=0 \\
\left(\varepsilon_{q}+P_{q}\right) & \left\{u_{q}^{\mu} \Delta_{q \eta \nu} u_{q ; \mu}^{\nu}\right\} \\
& -\Delta_{q \eta \tau} \frac{\partial P_{q}}{\partial \tau}+\frac{1}{\tau^{2}} \Delta_{q \eta \eta} \frac{\partial P_{q}}{\partial \eta}=0 .
\end{aligned}
$$

The equations (A2) and A3 are equivalent, therefore one has only one equation,

$$
\begin{aligned}
\left(\varepsilon_{q}+P_{q}\right) & \left\{\frac{\partial \alpha_{q}}{\partial \tau}+\frac{\tanh \left(\alpha_{q}-\eta\right)}{\tau} \frac{\partial \alpha_{q}}{\partial \eta}\right\} \\
& +\tanh \left(\alpha_{q}-\eta\right) \frac{\partial P_{q}}{\partial \tau}+\frac{1}{\tau} \frac{\partial P_{q}}{\partial \eta}=0,(\mathrm{~A} 4)
\end{aligned}
$$

which is the Eq. (27). Since the four divergence of the fluid velocity $u_{q ; \mu}^{\mu}$ is given by

$$
u_{q ; \mu}^{\mu}=\sinh \left(\alpha_{q}-\eta\right) \frac{\partial \alpha_{q}}{\partial \tau}+\frac{1}{\tau} \cosh \left(\alpha_{q}-\eta\right) \frac{\partial \alpha_{a}}{\partial \eta}
$$

the Eq. (23) can be written as

$$
\begin{aligned}
\cosh (\alpha-\eta) \frac{\partial \varepsilon_{q}}{\partial \tau} & +\frac{\sinh \left(\alpha_{q}-\eta\right)}{\tau} \frac{\partial \varepsilon_{q}}{\partial \eta} \\
+\left(\varepsilon_{q}+P_{q}\right)\left\{\sinh \left(\alpha_{q}-\eta\right) \frac{\partial \alpha_{q}}{\partial \tau}\right. & \\
& \left.+\frac{1}{\tau} \cosh \left(\alpha_{q}-\eta\right) \frac{\partial \alpha_{a}}{\partial \eta}\right\}=0
\end{aligned}
$$

leading immediately to

$$
\begin{aligned}
& \frac{\partial \varepsilon_{q}}{\partial \tau}+\frac{\tanh \left(\alpha_{q}-\eta\right)}{\tau} \frac{\partial \varepsilon_{q}}{\partial \eta} \\
& +\left(\varepsilon_{q}+P_{q}\right)\left\{\tanh \left(\alpha_{q}-\eta\right) \frac{\partial \alpha_{q}}{\partial \tau}+\frac{1}{\tau} \frac{\partial \alpha_{a}}{\partial \eta}\right\}=0
\end{aligned}
$$

which is Eq. (26).

\section{APPENDIX B: NUMERICAL METHOD USED}

For the purpose of numerical calculations we express Eqs.(26) and (27) in the form of finite difference equations :

$$
\begin{aligned}
& A_{1(j)}^{(n)}\left\{\frac{\varepsilon_{q(j)}^{(n+1)}-\frac{1}{2}\left[\varepsilon_{q(j+1)}^{(n)}+\varepsilon_{q(j-1)}^{(n)}\right]}{\Delta \tau}\right\}+A_{2(j)}^{(n)}\left\{\frac{\left.\varepsilon_{q(j+1)}^{(n)}-\varepsilon_{q(j-1)}^{(n)}\right\},}{2 \Delta \eta}\right\} \\
+ & A_{3(j)}^{(n)}\left\{\frac{\alpha_{q(j)}^{(n+1)}-\frac{1}{2}\left[\alpha_{q(j+1)}^{(n)}+\alpha_{q(j-1)}^{(n)}\right]}{\Delta \tau}\right\}+A_{4(j)}^{(n)}\left\{\frac{\alpha_{q(j+1)}^{(n)}-\alpha_{q(j-1)}^{(n)}}{2 \Delta \eta}\right\}=0
\end{aligned}
$$

and

$$
\begin{array}{r}
B_{1(j)}^{(n)}\left\{\frac{P_{q(j)}^{(n+1)}-\frac{1}{2}\left[P_{q(j+1)}^{(n)}+P_{q(j-1)}^{(n)}\right]}{\Delta \tau}\right\}+B_{2(j)}^{(n)}\left\{\frac{\left.P_{q(j+1)}^{(n)}-P_{q(j-1)}^{(n)}\right\},}{2 \Delta \eta}\right\} \\
+B_{3(j)}^{(n)}\left\{\frac{\alpha_{q(j)}^{(n+1)}-\frac{1}{2}\left[\alpha_{q(j+1)}^{(n)}+\alpha_{q(j-1)}^{(n)}\right]}{\Delta \tau}\right\}+B_{4(j)}^{(n)}\left\{\frac{\alpha_{q(j+1)}^{(n)}-\alpha_{q(j-1)}^{(n)}}{2 \Delta \eta}\right\}=0 .
\end{array}
$$


The subscript $(j)$ and superscript $(n)$ represent the corresponding grid number in the $\eta$ and $\tau$ space with grid spacings $\Delta \eta$ and $\Delta \tau$, respectively, i.e., with $\eta_{j}=j \Delta \eta$ and $\tau_{n}=\tau_{0}+n \Delta \tau$. The coefficients appearing in the above equations are defined in the following way:

$$
\begin{array}{ll}
A_{1(j)}^{(n)} \equiv 1, & B_{1(j)}^{(n)} \equiv v_{q(j)}^{(n)}, \\
A_{2(j)}^{(n)} \equiv\left[v_{q(j)}^{(n)}\right] / \tau_{n}, & B_{2(j)}^{(n)} \equiv 1 / \tau_{n}, \\
A_{3(j)}^{(n)} \equiv\left(\varepsilon_{q(j)}^{(n)}+P_{q(j)}^{(n)}\right)\left[v_{q(j)}^{(n)}\right], & B_{3(j)}^{(n)} \equiv\left(\varepsilon_{q(j)}^{(n)}+P_{q(j)}^{(n)}\right), \\
A_{4(j)}^{(n)} \equiv\left(\varepsilon_{q(j)}^{(n)}+P_{q(j)}^{(n)}\right) / \tau_{n}, & B_{4(j)}^{(n)} \equiv\left(\varepsilon_{q(j)}^{(n)}+P_{q(j)}^{(n)}\right)\left[v_{q(j)}^{(n)}\right] / \tau_{n} .
\end{array}
$$

Introducing now notation,

$$
c_{s(j)}^{2(n)} \equiv \frac{P_{q(j)}^{(n)}}{\varepsilon_{q(j)}^{(n)}},
$$

where $c_{s(j)}^{2(n)}$ is function of $\varepsilon_{q(j)}^{(n)}$ (due to the equation of state $P_{q}\left(\varepsilon_{q}\right)$ ), one can rewrite above two equations in the following form:

$$
\begin{aligned}
{\left[A_{1(j)}^{(n)}\right] } & \varepsilon_{q(j)}^{(n+1)}+\left[A_{3(j)}^{(n)}\right] \alpha_{q(j)}^{(n+1)} \\
& -\frac{1}{2}\left[A_{1(j)}^{(n)}-A_{2(j)}^{(n)} \frac{\Delta \tau}{\Delta \eta}\right] \varepsilon_{q(j+1)}^{(n)}-\frac{1}{2}\left[A_{1(j)}^{(n)}+A_{2(j)}^{(n)} \frac{\Delta \tau}{\Delta \eta}\right] \varepsilon_{q(j-1)}^{(n)} \\
& -\frac{1}{2}\left[A_{3(j)}^{(n)}-A_{4(j)}^{(n)} \frac{\Delta \tau}{\Delta \eta}\right] \alpha_{q(j+1)}^{(n)}-\frac{1}{2}\left[A_{3(j)}^{(n)}+A_{4(j)}^{(n)} \frac{\Delta \tau}{\Delta \eta}\right] \alpha_{q(j-1)}^{(n)}=0
\end{aligned}
$$

and

$$
\begin{aligned}
c_{s(j)}^{2(n+1)} & {\left[B_{1(j)}^{(n)}\right] \varepsilon_{q(j)}^{(n+1)}+\left[B_{3(j)}^{(n)}\right] \alpha_{q(j)}^{(n+1)} } \\
& -\frac{c_{s(j+1)}^{2(n)}}{2}\left[B_{1(j)}^{(n)}-B_{2(j)}^{(n)} \frac{\Delta \tau}{\Delta \eta}\right] \varepsilon_{q(j+1)}^{(n)}-\frac{c_{s(j-1)}^{2(n)}}{2}\left[B_{1(j)}^{(n)}+B_{2(j)}^{(n)} \frac{\Delta \tau}{\Delta \eta}\right] \varepsilon_{q(j-1)}^{(n)} \\
& -\frac{1}{2}\left[B_{3(j)}^{(n)}-B_{4(j)}^{(n)} \frac{\Delta \tau}{\Delta \eta}\right] \alpha_{q(j+1)}^{(n)}-\frac{1}{2}\left[B_{3(j)}^{(n)}+B_{4(j)}^{(n)} \frac{\Delta \tau}{\Delta \eta}\right] \alpha_{q(j-1)}^{(n)}=0 .
\end{aligned}
$$

Eliminating $\alpha_{q(j)}^{(n+1)}$ from the above two equations, one obtains

$$
F_{10(j)}^{(n)} \varepsilon_{q(j)}^{(n+1)}+F_{1+(j)}^{(n)} \varepsilon_{q(j+1)}^{(n)}+F_{0-(j)}^{(n)} \varepsilon_{q(j-1)}^{(n)}+G_{0+(j)}^{(n)} \alpha_{q(j+1)}^{(n)}+G_{0-(j)}^{(n)} \alpha_{q(j-1)}^{(n)}=0,
$$

where

$$
\begin{aligned}
& F_{10(j)}^{(n)} \equiv 1-c_{s(j)}^{2(n+1)}\left[v_{q(j)}^{(n)}\right]^{2}, \\
& F_{0+(j)}^{(n)} \equiv-\frac{1}{2}\left(1-c_{s(j+1)}^{2(n)}\left[v_{q(j)}^{(n)}\right]^{2}\right)+\frac{1}{2}\left(1-c_{s(j+1)}^{2(n)}\right) \frac{\left[v_{q(j)}^{(n)}\right]}{\tau_{n}} \frac{\Delta \tau}{\Delta \eta}, \\
& F_{0-(j)}^{(n)} \equiv-\frac{1}{2}\left(1-c_{s(j-1)}^{2(n)}\left[v_{q(j)}^{(n)}\right]^{2}\right)-\frac{1}{2}\left(1-c_{s(j-1)}^{2(n)}\right) \frac{\left[v_{q(j)}^{(n)}\right]}{\tau_{n}} \frac{\Delta \tau}{\Delta \eta}, \\
& G_{0+(j)}^{(n)} \equiv+\frac{1}{2}\left(1-\left[v_{q(j)}^{(n)}\right]^{2}\right)\left(\varepsilon_{q(j)}^{(n)}+P_{q(j)}^{(n)}\right) \frac{1}{\tau_{n}} \frac{\Delta \tau}{\Delta \eta}, \\
& G_{0-(j)}^{(n)} \equiv-\frac{1}{2}\left(1-\left[v_{q(j)}^{(n)}\right]^{2}\right)\left(\varepsilon_{q(j)}^{(n)}+P_{q(j)}^{(n)}\right) \frac{1}{\tau_{n}} \frac{\Delta \tau}{\Delta \eta} .
\end{aligned}
$$

One can now find $\varepsilon_{q(j)}^{(n+1)}$ by solving the non-linear Eq. (BB7). For $v_{q(j)}^{(n)}=0$ (i.e., for the scaling case where $\eta=\alpha$ ) one obtains

$$
\varepsilon_{q(j)}^{(n+1)}-\varepsilon_{q(j)}^{(n)}+\frac{\varepsilon_{q(j)}^{(n)}+P_{q(j)}^{(n)}}{\tau_{n}} \Delta \tau=0
$$


where relations $\alpha_{q(j+1)}^{(n)}-\alpha_{q(j-1)}^{(n)}=2 \Delta \eta$ and $\frac{1}{2}\left[\varepsilon_{q(j+1)}^{(n)}+\varepsilon_{q(j-1)}^{(n)}\right]=\varepsilon_{q(j)}^{(n)}$ were used. After finding $\varepsilon_{q(j)}^{(n+1)}$ one can find $\alpha_{q(j)}^{(n+1)}$ by using the following recurrence formula:

$$
\begin{aligned}
\alpha_{q(j)}^{(n+1)}= & \frac{1}{2}\left[\alpha_{q(j+1)}^{(n)}+\alpha_{q(j-1)}^{(n)}\right]-\frac{1}{2}\left[\alpha_{q(j+1)}^{(n)}-\alpha_{q(j-1)}^{(n)}\right] \frac{v_{q(j)}^{(n)}}{\tau_{n}} \frac{\Delta \tau}{\Delta \eta} \\
& +\frac{v_{q(j)}}{2}\left[\frac{c_{s(j+1)}^{2(n)}}{1+c_{s(j)}^{2(n)}} \frac{\varepsilon_{q(j+1)}^{(n)}}{\varepsilon_{q(j)}^{(n)}}+\frac{c_{s(j-1)}^{2(n)}}{1+c_{s(j)}^{2(n)}} \frac{\varepsilon_{q(j-1)}^{(n)}}{\varepsilon_{q(j)}^{(n)}}-2 \frac{c_{s(j)}^{2(n+1)}}{1+c_{s(j)}^{2(n)}} \frac{\varepsilon_{q(j)}^{(n+1)}}{\varepsilon_{q(j)}^{(n)}}\right] \\
& -\frac{1}{2}\left[\frac{c_{s(j+1)}^{2(n)}}{1+c_{s(j)}^{2(n)}} \frac{\varepsilon_{q(j+1)}^{(n)}}{\varepsilon_{q(j)}^{(n)}}-\frac{c_{s(j-1)}^{2(n)}}{1+c_{s(j)}^{2(n)}} \frac{\varepsilon_{q(j-1)}^{(n)}}{\varepsilon_{q(j)}^{(n)}}\right] \frac{1}{\tau_{n}} \frac{\Delta \tau}{\Delta \eta}
\end{aligned}
$$

\section{APPENDIX C: INADEQUACY OF THE SIMPLE EXPANSION IN $|q-1|$}

From the previous experience in applying $q$-statistics to multiparticle production [10, 11, 12, 13, 14, 15] we know that $|q-1|<1$. It seems then natural to argue that (see, for example, 11,50$]$ ) one could simply expand $f_{q}(x, p)=\left[1-(1-q) \frac{p^{\mu} u_{\mu}}{k_{B} T(x)}\right]^{1 /(1-q)}$ from Eq. (14) in $z=1-q$, retaining only terms linear in $z$ and get:

$$
\begin{aligned}
f_{q}(x, p) & =f(z)=[1-z \cdot A]^{\frac{1}{z}} \equiv\left[\frac{1}{z} \ln (1-z \cdot A)\right] \\
& \simeq f(z=0)+\left.z \cdot \frac{d f(z)}{d z}\right|_{z=0}
\end{aligned}
$$

(here $A=A(x, p)=\frac{p^{\mu} u_{\mu}(x)}{k_{B} T}$ and the arguments $(x, p)$ are suppressed for clarity).

However, such expansion can only be performed under some conditions, which we shall clarify in what follows. It is straightforward to show that to gets first step of expansion,

$$
\begin{aligned}
f(z) \simeq & \exp \left[-A \cdot\left(1+\frac{A}{2} \cdot z+\ldots\right)\right] \\
& =\exp [-A] \cdot \exp \left[-\frac{A^{2}}{2} \cdot z-\ldots\right],
\end{aligned}
$$

it is necessary that

$$
z \cdot A(x, p)<1 .
$$

The second step needed is to additionally expand the exponent and this requires

$$
z \cdot A^{2}(x, p)<2 .
$$

When this is satisfied, one finally gets $f_{q}(x, p)$ in terms of $f_{q=1}(x, p)$ only,

$$
f_{q}(x, p) \simeq f_{q=1}(x, p)+(1-q)\left[1-\frac{A^{2}(x, p)}{2}\right] f_{q=1}(x, p) .
$$

At first this procedure looks very promising because using it one gets

$$
\mathcal{T}_{q}^{\mu \nu} \equiv \mathcal{T}_{q=1}^{\mu \nu}+(q-1) \tau_{q}^{\mu \nu},
$$

where $\mathcal{T}_{q=1}$ is the usual energy-momentum tensor for the equilibrium of the Boltzmann-Gibbs statistics, i.e. the one usually used when describing an ideal fluid,

$$
\mathcal{T}_{q=1}^{\mu \nu} \equiv \frac{g}{(2 \pi)^{3}} \int \frac{d^{3} p}{p^{0}} p^{\mu} p^{\nu} \exp \left(-\frac{p \cdot u}{T}\right),
$$

whereas the nonextensive correction tensor $\tau_{q}^{\mu \nu}$ is given by

$$
\begin{aligned}
& \tau_{q}^{\mu \nu} \equiv \frac{g}{(2 \pi)^{3}} \int \frac{d^{3} p}{p^{0}} p^{\mu} p^{\nu} . \\
& \cdot \exp \left(-\frac{p \cdot u}{T}\right)\left[-\left(\frac{p \cdot u}{T}\right)+\frac{1}{2}\left(\frac{p \cdot u}{T}\right)^{2}\right]
\end{aligned}
$$

However, in our case condition (C4) would impose too severe constraints on the allowed $q$ and the region of phase space, $p$ and $x$, considered rendering this approximation rather unpractical for our purposes.

\section{APPENDIX D: DERIVATION OF EQ. (71) AND $\eta / s$, EQ. (73).}

The entropy production is given by

$$
\sigma_{\text {full } ; \mu}^{\mu}=\left[\xi \Psi^{\mu}\right]_{; \mu}=\frac{\partial_{\mu} \xi}{\xi} \xi \Psi^{\mu}+\xi \Psi_{; \mu}^{\mu}
$$

where

$$
\begin{aligned}
\Psi^{\mu} & =-\frac{W^{\nu} W_{\nu}}{3 \Pi T} u^{\mu}+\frac{W_{\nu}}{2 \Pi T} \pi^{\mu \nu} \\
& =\frac{-1}{6 \Pi T}\left\{2 W^{\nu} W_{\nu} u^{\mu}-3 W_{\nu} \pi^{\mu \nu}\right\} .
\end{aligned}
$$

Then,

$$
\Psi_{; \mu}^{\mu}=\left(\frac{\partial_{\mu} \Pi}{\Pi}+\frac{\partial_{\mu} T}{T}\right) \Psi^{\mu}+\frac{-1}{6 \Pi T} \psi_{; \mu}^{\mu},
$$


where $\psi^{\mu} \equiv 2 W^{\nu} W_{\nu} u^{\mu}-3 W_{\nu} \pi^{\mu \nu}$. The $\psi_{; \mu}^{\mu}$ is explicitly written as

$$
\begin{aligned}
\psi_{; \mu}^{\mu} & =\left\{2 W^{\nu} W_{\nu} u^{\mu}-3 W_{\nu} \pi^{\mu \nu}\right\}_{; \mu} \\
& =\left[4 W_{\nu ; \mu} u^{\mu}+2 W_{\nu} u_{; \mu}^{\mu}-3 \pi_{\nu ; \mu}^{\mu}\right] W^{\nu}+\left[-3 W_{\nu ; \mu}\right] \pi^{\mu \nu}
\end{aligned}
$$

and

$$
\begin{aligned}
& \frac{-1}{6 \Pi T} \psi_{; \mu}^{\mu}= \\
& \quad \frac{-1}{\Pi}\left[\frac{2}{3} W_{\nu ; \mu} u^{\mu}+\frac{1}{3} W_{\nu} u_{; \mu}^{\mu}-\frac{1}{2} \pi_{\nu ; \mu}^{\mu}\right] \frac{W^{\nu}}{T} \\
& \quad+\frac{-1}{\Pi}\left[-\frac{1}{2} W_{\nu ; \mu}\right] \frac{\pi^{\mu \nu}}{T} .
\end{aligned}
$$

Hence we obtain that

$$
\begin{aligned}
& \xi \Psi_{; \mu}^{\mu}=\xi(\left.\frac{\partial_{\mu} \Pi}{\Pi}+\frac{\partial_{\mu} T}{T}\right) \Psi^{\mu} \\
&-\frac{\xi}{\Pi}\left[\frac{2}{3} W_{\nu ; \mu} u^{\mu}+\frac{1}{3} W_{\nu} u_{; \mu}^{\mu}-\frac{1}{2} \pi_{\nu ; \mu}^{\mu}\right] \frac{W^{\nu}}{T} \\
&-\frac{\xi}{\Pi}\left[-\frac{1}{2} W_{\nu ; \mu}\right] \frac{\pi^{\mu \nu}}{T} \\
&=\xi\left(\frac{\partial_{\mu} \Pi}{\Pi}+\frac{\partial_{\mu} T}{T}\right) \Psi^{\mu}-Y_{\nu} \frac{W^{\nu}}{T}+Z_{\mu \nu} \frac{\pi^{\mu \nu}}{T}
\end{aligned}
$$

Finally one arrives at

$$
\begin{aligned}
\sigma_{\text {full; } \mu}^{\mu}= & {\left[\xi \Psi^{\mu}\right]_{; \mu} } \\
= & \frac{\xi}{\Pi}\left(\frac{\partial_{\mu} \xi}{\xi}+\frac{\partial_{\mu} \Pi}{\Pi}+\frac{\partial_{\mu} T}{T}\right) \Pi \Psi^{\mu} \\
& -Y_{\nu} \frac{W^{\nu}}{T}+Z_{\mu \nu} \frac{\pi^{\mu \nu}}{T} \\
= & -X_{\mu} T \Psi^{\mu} \frac{\Pi}{T}-Y_{\nu} \frac{W^{\nu}}{T}+Z_{\mu \nu} \frac{\pi^{\mu \nu}}{T} \\
= & -X_{\mu}\left(w u^{\mu}\right) \frac{\Pi}{T}-\tilde{Y}_{\nu} \frac{W^{\nu}}{T}+Z_{\mu \nu} \frac{\pi^{\mu \nu}}{T} .
\end{aligned}
$$

This is Eq. (71). Using now $\pi^{\mu \nu} W_{\nu}=-2 \Pi W^{\mu}$ one gets that

$$
\begin{aligned}
\sigma_{\text {full } ; \mu}^{\mu} & =-X_{\mu}\left(w u^{\mu}\right) \frac{\Pi}{T}-\tilde{Y}_{\nu} \frac{W^{\nu}}{T}+Z_{\mu \nu} \frac{\pi^{\mu \nu}}{T} \\
& =-X_{\mu}\left(w u^{\mu}\right) \frac{\Pi}{T}+\left[\frac{\tilde{Y}_{\nu} W_{\nu}}{2 \Pi T}+Z_{\mu \nu}\right] \frac{\pi^{\mu \nu}}{T} \\
& =-X_{\mu}\left(w u^{\mu}\right) \frac{\Pi}{T}+\tilde{Z}_{\mu \nu} \frac{\pi^{\mu \nu}}{T}
\end{aligned}
$$

which is Eq. (73).
[1] B. Müller, Nucl. Phys. A774, 433 (2006) and references therein; cf. also M. Gyulassy, L. McLerran, Nucl. Phys. A750, 30 (2005); I. Vitev, Int.J. Mod. Phys. A20, 3777 (2005); R.D. Pisarski, Braz. J. Phys. 36, 122 (2006) and references therein.

[2] G. Wilk and Z. Włodarczyk, Phys. Rev. Lett. 84, 2770 (2000); T.S. Biro and A. Jakovác, Phys. Rev. Lett. 94, 132302(2005).

[3] G. Wilk and Z. Włodarczyk, Chaos, Solitons and Fractals 13/3, 581 (2001).

[4] Like, for example, the flow phenomenon or decay of resonances, see B. Müller and J.L. Nagle, Ann. Rev. Nuc. Part. Sci. 56, 93 (2006) and references therein.

[5] C.Tsallis, J. Stat. Phys. 52, 479 (1988), Braz. J. Phys. 29, 1 (1999), Physica A340, 1 (2004) and Physica A344, 718 (2004) and references therein. See also Nonextensive Statistical Mechanics and its Applications, S. Abe and Y. Okamoto (Eds.), Lecture Notes in Physics LPN560, Springer (2000).

[6] T.S. Biró and G. Purcsel, Phys. Rev. Lett. 95162302 (2005).

[7] T.S. Biró and G. Kaniadakis, Eur. Phys. J. B50, 3 (2006) and references therein.

[8] T.S. Biró and G. Purcsel, Phys. Lett. A372, 1174 (2008).

[9] T. Sherman and J. Rafelski, Lecture Notes in Physics 633 (2004) 377.

[10] I. Bediaga, E.M. Curado and J.M.de Miranda, Physica A286, 156 (2000); T. Wibig and I. Kurp, Int. J. High Energy Phys. 0312, 039 (2003).

[11] W.M. Alberico, A. Lavagno and P. Quarati, Eur. Phys. J. C12, 499 (2000).
[12] O.V. Utyuzh, G. Wilk and Z. Włodarczyk, J. Phys. G26, L39 (2000); G. Wilk and Z. Włodarczyk, Nucl. Phys. (Proc. Suppl.) 75A, 191 (1999); Physica A305, 227 (2002); M. Rybczyński, Z. Włodarczyk and G. Wilk, Nucl. Phys. (Proc. Suppl.) B97, 81 (2001) and B122, 325 (2003).

[13] F.S. Navarra, O.V. Utyuzh, G. Wilk and Z. Włodarczyk, Phys. Rev. D67, 114002 (2003); Physica A344, 568 (2004); Nukleonika 49 (Supplement 2), S19 (2004).

[14] F.S. Navarra, O.V. Utyuzh, G. Wilk and Z. Włodarczyk, Physica A340, 467 (2004).

[15] M. Biyajima, M. Kaneyama, T. Mizoguchi and G. Wilk, Eur. Phys. J. C40, 243 (2005); M. Biyajima, T. Mizoguchi, N. Nakajima, N. Suzuki, and G. Wilk, Eur. Phys. J. C48, 593 (2006).

[16] T. Osada, O.V. Utyuzh, G. Wilk and Z. Włodarczyk, Europ. Phys. J. B50, 7 (2006).

[17] A. Lavagno, Phys. Lett. A301, 13 (2002).

[18] W.M. Alberico, P. Czerski, A. Lavagno, M. Nardi and V. Somá, Physica A387, 467 (2008).

[19] F.S. Navarra, O.V. Utyuzh, G. Wilk and Z. Włodarczyk, Nuovo Cim. 24C, 725 (2001).

[20] G. Wilk and Z. Włodarczyk, Physica A376, 279 (2007).

[21] For the most recent reviews see: P. Huovinen, P.V. Ruuskanen, Ann. Rev. Nucl. Part. Phys. 56, 163 (2006); T. Hirano, J.Phys. G30, S845 (2004); T. Hirano, Y. Nara, J.Phys. G31, S1 (2005); Y. Hama, T. Kodama and O. Socolowski Jr., Braz.J.Phys. 35, 24 (2005) and references therein.

[22] B.M. Boghosian, Braz.J.Phys. 29, 91 (1999); F.Q. Potiguar, U.M.S. Costa, Physica A303, 457 (2002). 
[23] C. Eckart, Phys. Rev. 58, 919 (1940).

[24] W. Israel, Ann. Phys. (N.Y.) 100, 310 (1976); J. M. Stewart, Proc. R. Soc. London, Ser. A 357, 59 (1977); W. Israel and J. M. Stewart, Ann Phys. (N.Y.) 118, 341 (1979).

[25] A. Muronga, Phys. Rev. C 69, 034903 (2004) and Phys. Rev. Lett. 88, 062302 (2002); A. Muronga and D.H. Rischke, arXiv:nucl-th/0407114.

[26] R. Baier, P. Romatschke and U. A. Wiedmann, Phys. Rev. C 73, 064903 (2006); R. Baier and P. Romatschke, Eur. Phys. J. C 51, 677 (2007); P. Romatschke, Eur. Phys. J. C 52, 203 (2007); P. Romatschke and U. Romatschke, Phys. Rev. Lett. 99, 17230 (2007).

[27] See, for example, K. Tsumura and T. Kunihiro, arXiv:0709.3645 [nucl-th] and references therein.

[28] W. A. Hiscock and L. Lindblom, Ann. Phys. (N.Y.) 151, 466 (1983); Phys. Rev. D31, 725 (1985); D35, 3723 (1987).

[29] A.K. Chaudhuri, Phys. Rev. C 74, 044904 (2006) and arXiv:0704.0134 [nucl-th].

[30] U. Heinz, H. Song and A.K. Chaudhuri, Phys. Rev. C 73, 034904 (2006); H. Song and U. Heinz, arXiv:0712.3715 [nucl-th].

[31] T. Koide, G.S. Denicol, Ph. Mota and T. Kodama, Phys. Rev. C 75, 034909 (2007).

[32] A. Dumitru, E. Molnár and Y. Nara, Phys. Rev. C 76, 024910 (2007).

[33] T. Hirano and K. Tsuda, Phys. Rev. C66, 054905 (2002).

[34] D. Teaney, Phys. Rev. C68, 034913 (2003).

[35] A. K. Chaudhuri, Viscous fluid dynamics in Au+Au collisions at RHIC, arXiv:0801.3180 and references therein.

[36] P. Kovtun, D. T. Son and A. O. Starinets, Phys. Rev. Lett. 94, 111601 (2005). For the most recent review of this subject see D. T. Son ans A. O. Starinet, Annu. Rev. Nucl. Part. Sci. 57, 95 (2007) and references therein.

[37] J.A.S. Lima, R. Silva and A.R. Plastino, Phys. Rev. Lett. 86, 2983 (2001).

[38] S. Abe, Physica A368, 430 (2006).

[39] L.M. Satarow, I.N. Mishustin, A.V. Merdeev and H. Stöcker, Phys. Rev. C75, 024903 (2007).

[40] A. Drago, A. Lavagno and P. Quarti, Physica A344, 472 (2004).

[41] St. Mrówczyński, Acta Phys. Polon. B37, 427 (2006)

[42] P. Castorina, D. Kharzeev and H. Satz, Eur. Phys. J. C2, 187 (2007).

[43] T. Hirano, Phys. Rev. C65, 011901(R) (2002).

[44] R.C. Hwa, Phys. Rev. D10, 2260 (1974); J.D. Bjorken, Phys. Rev. D27, 140 (1983).

[45] L.D.Landau, Izv.Akad.Naul.SSSR, Ser.Fiz. 17, 51 (1953).

[46] I.G. Bearden et.al. (BRAHMS Collaboration), Phys. Rev. Lett. 93, 102301 (2004).

[47] F. Cooper and G. Frye, Phys. Rev. D10, 186 (1974).

[48] B.I. Abelev et al STAR Collaboration, Phys. Rev. Lett. 97, 152301 (2006).

[49] J.G. Bearden et al BRAHMS Collaboration, Phys. Rev. Lett. 94, 162301 (2005).

[50] C.Beck, Physica A286, 164 (2000).

[51] A.Majumder, B.Müler and X.-N. Wang, Phys. Rev. Lett. 99, 192301 (2007); D.Molnar and M.Gyulassy, Nucl. Phys. A697, 495 (2002).

[52] R. Andrade, F. Grassi, Y. Hama, T. Kodama and O. Socolowski Jr., Phys. Rev. Lett. 97, 202302 (2006); Y. Hama, R.Peterson, G. Andrade, F. Grassi, W.-L. Qian,
T. Osada, C.E. Aguiar and T. Kodama, arXiv:0711.4544 to appear in Phys. Atom. Nucl. (2008).

[53] S. Vogel, G. Torrieri and M. Bleicher, nucl-th/0703031 and references therein.

[54] Ch.G.Weert, Annals. Phys. 140, 133 (1982); W. Schoenmaker and M. Ichiyanagi, Covariant derivation of relativistic hydrodynamics, Univ. Libre de Bruxelles preprint, November 1987.

[55] O.V. Utyuzh, G. Wilk and Z. Włodarczyk; Acta Phys. Hung. A -Heavy Ion Phys. 25, 65 (2006).

[56] G. Wilk and Z. Włodarczyk, Phys. Rev. D43, 794 (1991).

[57] The situation in which $q<1$ was analyzed in [19]. As discussed in [3], interpretation in terms of fluctuations is not clear now. Instead it was shown that in this case the first role of the parameter $q$ is to restrict the allowed phase space and $q<1$ reflects the fact that only a fraction $K$ (called inelasticity) of the initially available energy is used for the production of secondaries, the remaining $1-K$ part is to be found in the so called leading particles. As a result one gets $\exp _{q<1}(-X)$ distribution with $X$ limited to the $X>1 /(1-q)$ region only.

[58] Actually, these are precisely the same fluctuations that lead to the Negative Binomial form of the observed multiplicity distributions, $P(n ;\langle n\rangle ; k)$, with its characteristic parameter $k$ given by $k=1 /\left(q_{\mathrm{L}}-1\right)$ [13] (in this case one can also speak about fluctuations in the so called partition temperature $T_{p t}=E /\langle n\rangle$, see [20].

[59] In this case $q$ measures fluctuation of the temperature $T$ as given by the specific heat parameter $C$ and $q_{\mathrm{T}}-1=C$ 2, [15]. As such it should be inversely proportional to the volume of the interaction region, this effect is indeed observed [15].

[60] Notice that our analysis of multiparticle production using a $q$-hydrodynamical model differs substantially from previous applications of $q$ statistics presented in 10, 11, 12, 13, 14, 15, 16, 17, 18] because now the local thermal equilibrium (in its $q$-version) is superimposed on the longitudinal flow. It is therefore not clear a priori which $q$ should enter at which step of the collision process. We plan to discuss this subject elsewhere.

[61] Notice that the normalization $Z_{q}$ and (unnormalized) energy density $T_{q}^{\mu \nu}$ are conserved independently. Therefore our further consideration will be concentrated only on getting $f_{q}(x, p)$. However, the $q$-dependent normalization is important when analyzing particle distribution functions because it couples the widths of distributions (as given by $f_{q}$ ) with their heights (as given by $Z_{q}$, cf. [13]).

[62] One should be aware of the fact that there is still an ongoing discussion on the meaning of the temperature in nonextensive systems. However, the small values of the parameter $q$ deduced from data allow us to argue that, to first approximation, $T$ can be regarded as the hadronizing temperature in such a system. One must only remember that in general what we study here is not so much the state of equilibrium but rather some kind of stationary state. For a thorough discussion of the temperature of nonextensive systems, see [38].

[63] The covariant derivatives of the vector $u^{\mu}$ and tensor $g^{\mu \nu}$ are defined by using the Christoffel symbol $\Gamma_{\lambda \mu}^{\nu} \equiv$ $\frac{1}{2} g^{\nu \sigma}\left(\partial_{\mu} g_{\sigma \lambda}+\partial_{\lambda} g_{\sigma \mu}-\partial_{\sigma} g_{\lambda \mu}\right)$ and are equal to $u_{; \mu}^{\nu}=$ $\partial_{\mu} u^{\nu}+\Gamma_{\lambda \mu}^{\nu} u^{\lambda} \quad$ and $g_{; \mu}^{\mu \nu}=\partial_{\mu} g^{\mu \nu}+\Gamma_{\sigma \mu}^{\mu} g^{\sigma \nu}+\Gamma_{\sigma \mu}^{\nu} g^{\mu \sigma}$, respectively.

[64] See, for example, review by [41] and references therein. 
We mention at this point that the so called kinematic thermalization used here in which equilibration of energies is due to the collisions, has been recently contrasted with the so called stochastic thermalization based on the process of erasing of memory of the initial state resulting in a state of maximal entropy and coinciding with the above thermal equilibrium state, see [42] and references therein.

[65] Notice that, in principle, both the $N_{\text {part }}$ and the $\Delta E$ are also fluctuating quantities but we shall not consider these fluctuations here. One can argue that they are to some extent accounted for by the nonextensive version of initial conditions considered here.

[66] For example, in [9] it was a random distortions of energy and momentum conservation caused by the surrounding system which resulted in emergence of some nonextensive equilibrium. In [6, 7] the two-body energy composition is replaced by generalized energy sum $h\left(E_{1}, E_{2}\right)$ (assumed to be associative) which is not necessarily the simple addition and which contains contributions stemming from the pair interaction. It turns out that under quite general assumptions about the function $h$ the division of the total energy among free particles can be done. Different forms of function $h$ lead to different forms of entropy formula, among which one encounters the known Tsallis form as well. The origin of this kind of thinking can be traced to analysis of the $q$-Hagedorn model in [50].

[67] Notice that whereas the time evolution of $\Pi$ is controlled by $q$-hydrodynamics (via the respective time dependencies of $\varepsilon_{q}, P_{q}$ and $x$ ) its form is determined by the assumed constraints which must assure that the local entropy production in the standard $2^{\text {nd }}$ order hydrodynamic theory [24, 25] is never negative.

[68] The fact that we obtained non-zero coefficients $\beta_{i=1,2,3}$ and couplings $\alpha_{i=0,1}$ for dissipative flux of Eq. (60) as found in Eq. (68) means that $d$-hydrodynamics obtained via NexDC from $q$-hydrodynamics accounts for all second order terms. One may conclude than it seems that such $d$ hydrodynamics with full order entropy current has global causality (over a distance scale given by relaxation time). However, question whether NexDC violates causality remains so far unsettled. 